\title{
A STUDY OF THE INCIDENCE OF DISEASE IN A WHALING EXPEDITION TO THE ANTARCTIC PELAGIC WHALING GROUNDS 1946-7
}

\author{
BY \\ R. A. M. CASE \\ From the Royal Naval Physiological Laboratory
}

Whaling is at present an occupation of some economic importance; and the present world fat shortage, coupled with a growing interest in the utilization of whale meat for human food, makes it likely that more people will be so engaged during the next few years. At present, about ten or twelve fleets, each with a complement of some five hundred men, visit the Antarctic pelagic whaling grounds each year.

Each fleet may be expected to carry at least one medical officer, and presumably this has been done for many years, but there seems to be a scarcity of information in the literature about the medical aspects of whaling.

During the Balaena expedition of 1946-7, attention was drawn to the problem of sepsis amongst certain classes of workers, and the ship's Medical Officer, Dr. Jamis Hutchison, consulted me on many occasions about this problem.

The Balaena expedition was probably unique in whaling history in that it had on board a laboratory in which biochemical, physiological, and bacteriological investigations could be undertaken. The bacteriology was of limited scope, for when the expedition sailed it was not realized that one of the major problems confronting the research team would be bacteriological in nature, and the laboratory had to be partly transformed at sea. Nonetheless, certain findings of some importance did emerge from these bacteriological studies (Case, 1947).

Since attention had been drawn to the problems of sepsis, it was considered worth while analysing the whole of the available hospital data from the Balaena sick bay, and this information was willingly placed at my disposal by Dr. Hutchison. The following work is the result of this analysis, with a discussion of the possible implications, and a report of some of the medical work carried out in the laboratory. It is essentially a "post-mortem" study of the data, not the outcome of a piece of planned research, so many shortcomings are apparent in the method of collecting and classifying the material, but the study is offered as a "pilot" investigation in the hope that it will be followed up by subsequent investigators, and as a record of data for subsequent comparison.

\section{The Technique of Whaling}

Before embarking upon a consideration of the data, it will be convenient to outline the main operations that took place during the whaling expedition.

The voyage lasted for thirty-three weeks. Eleven weeks of this time were spent in reaching and returning from the whaling grounds, and twenty-two weeks in whaling. Four of these weeks were devoted to chasing sperm whales (Physeter cotodon), and the remainder of the time to taking baleen whales (Balaenoptera musculus and B. physalus, the Blue and Fin whales). These twenty-two weeks will, for the purpose of this communication, be treated as one homogeneous period of whaling.

The whaling took place in an area lying roughly between $55^{\circ}$ and $65^{\circ}$ South and $40^{\circ}$ and $115^{\circ}$ East; and, altogether, about 2,500 whales were taken.

During the period of transit to and from the whaling grounds, the catchers assigned to the factory ship were for the greater part of the time sailing independently, but a part of their complement (mainly gunners) was carried on the mother ship. During the whaling period the catchers, now carrying their full complement, would come alongside every two or three days for bunkering.

This meant that during the transit period, the facilities for hospital attendance of the catcher crews would be in no way comparable with those of the complement of the mother ship; and, even during the whaling period, there would be a tendency to more infrequent visits to hospital, or, perhaps, an 
attendance for only the less trivial complaints. Allowance will be made for this difference when considering the statistics of attendance.

The factory ship carried the ship's crew, which consisted of the officers, seamen, engineers, and firemen; and also the deck workers and factory operatives. The duties of the latter two groups were to deal with the whale carcase from the time that it was hauled on board until it was finally processed. A considerable degree of interchangeability between these two groups existed, and for the purposes of the present study it is fairer to treat them as one group.

Thus the total complement can be divided into three classes: the catcher crews, who spend most of their time away from the factory, and only handle whole whale carcases soon after death; the factory ship's crew, who are concerned almost entirely with the sailing and organization of the vessel; and the factory and deck workers, who have to handle, dismember, and process the carcases.

The whale was hunted by the catchers, and was shot by a harpoon, the head of which carried a charge of black powder. When dead, the carcase was hauled alongside the catcher and inflated with air to increase its bouyancy. It was then either cast adrift, marked by a flag on the end of a pole, whilst the catcher sought fresh prey; or else made fast alongside and taken back to the factory ship. Here the carcase was towed astern until its turn came to be hauled up and processed.

A carcase might have been dead for anything up to thirty-six hours when processed, but a regulation forbids the Master to allow the catchers to take more whales than the factory can deal with in this space of time. There is an exception to this rule, however, for when a tanker came alongside, an event that occurred four or five times during the season, whales were used as fenders between the two vessels, and would sometimes remain there for several days. At the end of this time they were brought up and flensed, the blubber being utilized, and the remainder of the carcase was then jettisonned. The carcase was in an advanced state of decomposition by this time, and was both offensive to handle and a possible source of infection to those handling it.

The Dutch whaling expedition in 1946-7 made a practice of flensing their fender whales before they were used, and then allowing the flensed carcase to drift away after use. The handling of decomposed material thus was obviated. This method seems worthy of consideration and further investigation.

From its position astern, the whale was hauled up the slipway and flensed, a process in which the thick coating of blubber is sliced with special knives and then stripped from the carcase with wire ropes attached to power driven winches. The pieces of blubber were further subdivided and fed into boilers for the extraction of the oil. The rest of the carcase was then hauled farther forward and the meat cut from the bones. Usually this meat was fed into a further series of boilers to recover the oil, and the extracted residue dried to make meat meal. The bones were also cut into pieces and the oil extracted.

This description refers only to the general whaling routine and does not apply to the production of meat for human consumption, where special arrangements were made, and where special techniques are under consideration by both the whaling company and the appropriate authorities.

During these processes the spongy wooden deck became covered with a thick film of blood, fat and faeces, and since whaling went on by day and night, adequate washing down could only take place on the infrequent occasions when the weather or scarcity of whales interfered with whaling operations. The deck is also the deck-head of the factory, and in areas is kept quite warm by wild heat from below. Splinters from this deck could be a dangerous vector of infection.

\section{Hospital Organization}

The hospital was situated on board the factory ship, and usually there were two periods of surgery hours per day, morning and evening. In addition, accidents were treated at once at any time. Officially one medical officer was carried, but on this voyage assistance was available from a medically qualified member of the research team.

In my opinion it is highly desirable that an expedition of this nature should carry two medical officers, since in addition to the medical work of an ordinary ship's surgeon, there are the hazards of quite a large factory to cope with. Further, surgery has to be undertaken, and the employment of an untrained anaesthetist is very unsatisfactory if good results are to be obtained.

When a patient reported at the hospital, a card was filled in with the details of the sickness etc. For the purposes of the statistical analysis, one report of sickness counted as one attendance, irrespective of the number of subsequent visits for the same illness, and was, of course, recorded on the date of the first visit.

\section{Classification}

In the analysis, the diseases were grouped in an arbitrary manner in fourteen classifications. These are arranged for convenience in order of the magnitude of incidence, and will be referred to as columns 
one to fourteen. Occasionally a disease might appear in two classifications, for example suppurative otitis media would appear under the columns "suppurative lesions" and "diseases of the ear, nose, and throat." Since in these studies little emphasis is laid on the total morbidity figures, but on the column morbidity figures, this double entry does not invalidate the conclusions to be drawn.

\section{Classification of diseases}

The arbitrary classification adopted is shown in Table I. These groups will be referred to by the column number, both in the text and in subsequent tabulations.

TABLE I

The Classification of Disease arranged in descending ORDER OF MAGNITUDE OF INCIDENCE

\begin{tabular}{|c|c|}
\hline Column number & Disease classification \\
\hline 1 & Suppurative lesions \\
\hline 2. & $\begin{array}{l}\text { Traumatic conditions, including frac- } \\
\text { tures, without sepsis }\end{array}$ \\
\hline 3 & Diseases of the skin \\
\hline 4 & $\begin{array}{c}\text { Alimentary disorders other than } \\
\text { diarrhoea }\end{array}$ \\
\hline 5 & Diseases of the ear, nose, and throat \\
\hline 6 & "Rheumatic" conditions \\
\hline 7 & $\begin{array}{l}\text { Diseases (including foreign bodies) of } \\
\text { the eye }\end{array}$ \\
\hline 8 & Venereal diseases, all types \\
\hline 9 & Diseases of the upper respiratory tract \\
\hline 10 & Burns and scalds \\
\hline 11 & Dental conditions \\
\hline 12 & $\begin{array}{l}\text { Disorders of the central nervous } \\
\text { system (organic and functional) }\end{array}$ \\
\hline 13 & Diarrhoea \\
\hline 14 & $\begin{array}{l}\text { Miscellaneous disorders not included } \\
\text { in the above groups }\end{array}$ \\
\hline
\end{tabular}

\section{Classification of complement}

The classification has been made into three groups on the lines previously discussed, and is shown in Table II. These groups will be referred to subsequently as A, B, and C.
TABLE II

Classification of the Expedition's COMPlement

\begin{tabular}{c|l|c}
\hline Group & \multicolumn{1}{|c|}{ Description } & Number of men \\
\hline A & Deck and factory workers & 224 \\
\hline B & Factory ship's complement & 142 \\
\hline C & Catchers' complements & 174 \\
\hline & Total complement & 540 \\
\hline
\end{tabular}

Division of the season

The division is shown in Table III.

TABLE III

Division of SeAson

\begin{tabular}{c|c|c}
\hline Period & Description & Duration \\
\hline Transit & $\begin{array}{c}\text { Voyage to and from whaling } \\
\text { grounds }\end{array}$ & 11 weeks \\
\hline Whaling & $\begin{array}{c}\text { Time spent in catching whales } \\
\text { (Sperm, Blue, and Fin) }\end{array}$ & 22 weeks \\
\hline & Total voyage & 33 weeks \\
\hline
\end{tabular}

\section{Results}

Table IV sets out, week by week, the incidence of disease in each group for each column, and also the total morbidity by columns and by weeks. The total classified and unclassified morbidity is also given.

In order to facilitate comparison between the groups, these figures have also been computed as morbidity rate per thousand. These figures are given in extenso in Table $\mathrm{V}$, and the totals appended to Table IV.

Figs. 1 to 13 show the weekly morbidity rate per thousand for each group as superimposed histograms.

Table VI sets out the weekly means with standard deviations of the morbidity rate per thousand for each column and group for each period, and also shows the difference of the means of each period for groups A and B. The parameters of significance, using " Student's" (1908) " $t$ " test, are shown for these differences. No such comparison is attempted for group $\mathrm{C}$, since their facilities for hospital attendance were seriously curtailed during the transit period.

Table VII shows the mean of the differences (not the differences of the means) of the weekly morbidity rate per thousand for groups $A$ and $B$ for both periods. Again $\mathrm{C}$ is not considered in these 
TABLE

InCidence of Classified Disease WeEk bY $\stackrel{\circ}{-}$

\begin{tabular}{|c|c|c|c|c|c|c|c|c|c|c|c|c|c|c|c|c|c|c|c|c|c|c|c|c|c|}
\hline \multirow[t]{2}{*}{ Period } & \multirow{2}{*}{$\begin{array}{c}\begin{array}{c}\text { Week } \\
\text { of } \\
\text { voyage }\end{array} \\
\text { Group }\end{array}$} & \multicolumn{3}{|c|}{$\begin{array}{c}\text { Suppurative } \\
\text { lesions } \\
1\end{array}$} & \multicolumn{3}{|c|}{$\begin{array}{c}\text { Trauma and } \\
\text { fractures } \\
\text { without sepsis } \\
2\end{array}$} & \multicolumn{3}{|c|}{$\begin{array}{c}\text { Diseases of } \\
\text { the skin } \\
3\end{array}$} & \multicolumn{3}{|c|}{$\begin{array}{c}\text { Non-diarrhoeic } \\
\text { alimentary } \\
\text { disorders } \\
4\end{array}$} & \multicolumn{3}{|c|}{$\begin{array}{c}\begin{array}{c}\text { Diseases of } \\
\text { ear, nose, and } \\
\text { throat }\end{array} \\
5\end{array}$} & \multicolumn{3}{|c|}{$\begin{array}{c}\text { " Rheumatic" } \\
\text { conditions } \\
6\end{array}$} & \multicolumn{3}{|c|}{$\begin{array}{c}\text { Diseases of } \\
\text { the eye } \\
7\end{array}$} & \multicolumn{3}{|c|}{$\begin{array}{c}\begin{array}{c}\text { Venereal } \\
\text { disease }\end{array} \\
8\end{array}$} \\
\hline & & A & B & C & A & B & C & A & B & C & A & B & C & A & B & C & A & B & C & A & B & C & A & B & C- \\
\hline Transit & $\begin{array}{l}1 \\
2 \\
3 \\
4 \\
5 \\
6\end{array}$ & $\begin{array}{l}1 \\
1 \\
1 \\
1 \\
1 \\
1\end{array}$ & \begin{tabular}{l|l}
0 & \\
1 & \\
1 & \\
2 & \\
1 & \\
3 & \\
\end{tabular} & $\begin{array}{l}0 \\
0 \\
0 \\
0 \\
1 \\
0\end{array}$ & \begin{tabular}{l|l}
0 & \\
1 & \\
1 & \\
2 & \\
6 & \\
1 &
\end{tabular} & $\begin{array}{l}1 \\
0 \\
4 \\
2 \\
2 \\
1\end{array}$ & $\begin{array}{l}0 \\
0 \\
0 \\
0 \\
0 \\
0 \\
0\end{array}$ & $\begin{array}{l}0 \\
1 \\
2 \\
6 \\
3 \\
2 \\
2\end{array}$ & $\begin{array}{l}0 \\
0 \\
1 \\
3 \\
3 \\
1 \\
1\end{array}$ & $\begin{array}{l}0 \\
0 \\
0 \\
4 \\
2 \\
0 \\
0\end{array}$ & $\begin{array}{l}0 \\
0 \\
0 \\
0 \\
0 \\
0\end{array}$ & $\begin{array}{l}0 \\
0 \\
2 \\
1 \\
0 \\
0\end{array}$ & \begin{tabular}{l|l}
0 & \\
1 & \\
0 & \\
0 & \\
0 & \\
0 &
\end{tabular} & $\begin{array}{l}0 \\
0 \\
0 \\
3 \\
2 \\
1\end{array}$ & $\begin{array}{l}1 \\
0 \\
0 \\
0 \\
4 \\
2\end{array}$ & $\begin{array}{l}0 \\
0 \\
1 \\
0 \\
0 \\
0\end{array}$ & $\begin{array}{l}0 \\
0 \\
0 \\
0 \\
1 \\
1\end{array}$ & $\begin{array}{l}0 \\
0 \\
1 \\
0 \\
1 \\
0\end{array}$ & \begin{tabular}{l|l}
0 & \\
0 & \\
0 & \\
0 & \\
1 & \\
1 &
\end{tabular} & $\begin{array}{l}0 \\
1 \\
0 \\
1 \\
2 \\
4\end{array}$ & \begin{tabular}{l|l}
0 & \\
0 & \\
1 & \\
0 & \\
1 & \\
1 &
\end{tabular} & $\begin{array}{l}0 \\
0 \\
0 \\
0 \\
0 \\
0\end{array}$ & $\begin{array}{l}0 \\
0 \\
4 \\
5 \\
2 \\
0\end{array}$ & $\begin{array}{l}0 \\
0 \\
0 \\
1 \\
1 \\
0\end{array}$ & $\begin{array}{l}1 \\
0 \\
0 \\
1 \\
1 . c \\
0 \\
0\end{array}$ \\
\hline Whaling & $\begin{array}{r}7 \\
8 \\
9 \\
10 \\
11 \\
12 \\
13 \\
14 \\
15 \\
16 \\
17 \\
18 \\
19 \\
20 \\
21 \\
22 \\
23 \\
24 \\
25 \\
26 \\
27 \\
28\end{array}$ & $\begin{array}{r}4 \\
5 \\
4 \\
9 \\
16 \\
5 \\
7 \\
7 \\
2 \\
9 \\
5 \\
8 \\
3 \\
4 \\
3 \\
5 \\
4 \\
4 \\
5 \\
2 \\
3\end{array}$ & $\begin{array}{l}3 \\
1 \\
0 \\
2 \\
3 \\
2 \\
1 \\
1 \\
0 \\
4 \\
0 \\
2 \\
0 \\
3 \\
1 \\
2 \\
1 \\
2 \\
4 \\
0 \\
0 \\
1\end{array}$ & $\begin{array}{l}1 \\
2 \\
0 \\
2 \\
0 \\
1 \\
0 \\
0 \\
0 \\
0 \\
1 \\
0 \\
0 \\
0 \\
0 \\
0 \\
0 \\
0 \\
0 \\
0 \\
1 \\
0\end{array}$ & $\begin{array}{l}2 \\
5 \\
3 \\
6 \\
4 \\
6 \\
1 \\
6 \\
2 \\
3 \\
3 \\
4 \\
5 \\
5 \\
4 \\
4 \\
8 \\
3 \\
6 \\
5 \\
6\end{array}$ & $\begin{array}{l}5 \\
2 \\
1 \\
2 \\
2 \\
0 \\
0 \\
0 \\
0 \\
2 \\
3 \\
1 \\
0 \\
1 \\
0 \\
1 \\
1 \\
0 \\
1 \\
2 \\
1\end{array}$ & $\begin{array}{l}0 \\
0 \\
0 \\
0 \\
0 \\
0 \\
1 \\
1 \\
1 \\
0 \\
1 \\
0 \\
1 \\
0 \\
0 \\
0 \\
0 \\
0 \\
0 \\
0 \\
0 \\
2\end{array}$ & \begin{tabular}{l|}
5 \\
5 \\
4 \\
6 \\
4 \\
4 \\
2 \\
5 \\
1 \\
2 \\
2 \\
5 \\
3 \\
5 \\
2 \\
0 \\
5 \\
4 \\
4 \\
5 \\
5 \\
3
\end{tabular} & $\begin{array}{l}2 \\
1 \\
1 \\
2 \\
0 \\
0 \\
0 \\
4 \\
0 \\
1 \\
0 \\
1 \\
0 \\
2 \\
0 \\
3 \\
1 \\
0 \\
2 \\
1 \\
0 \\
0\end{array}$ & $\begin{array}{l}0 \\
1 \\
0 \\
1 \\
0 \\
2 \\
0 \\
0 \\
0 \\
0 \\
0 \\
0 \\
0 \\
1 \\
0 \\
0 \\
0 \\
0 \\
0 \\
0 \\
1\end{array}$ & $\begin{array}{l}2 \\
2 \\
3 \\
3 \\
3 \\
2 \\
1 \\
3 \\
0 \\
3 \\
3 \\
2 \\
3 \\
4 \\
0 \\
0 \\
1 \\
2 \\
5 \\
5 \\
5 \\
3\end{array}$ & \begin{tabular}{l|}
1 \\
0 \\
0 \\
0 \\
1 \\
0 \\
0 \\
4 \\
6 \\
1 \\
0 \\
0 \\
2 \\
4 \\
0 \\
0 \\
2 \\
2 \\
2 \\
0 \\
1
\end{tabular} & \begin{tabular}{l|l}
0 \\
0 \\
1 \\
0 \\
1 \\
0 \\
1 \\
0 \\
2 \\
0 \\
0 \\
0 \\
2 \\
0 \\
0 \\
0 \\
0 \\
0 \\
0 \\
0 \\
2 \\
0
\end{tabular} & $\begin{array}{l}2 \\
2 \\
1 \\
6 \\
9 \\
4 \\
4 \\
2 \\
1 \\
1 \\
3 \\
1 \\
1 \\
0 \\
1 \\
0 \\
0 \\
2 \\
2 \\
1 \\
1 \\
0\end{array}$ & $\begin{array}{l}1 \\
1 \\
0 \\
1 \\
1 \\
0 \\
2 \\
2 \\
2 \\
0 \\
0 \\
0 \\
0 \\
0 \\
0 \\
0 \\
0 \\
0 \\
0 \\
1 \\
0 \\
0\end{array}$ & $\begin{array}{l}1 \\
0 \\
0 \\
0 \\
0 \\
0 \\
0 \\
0 \\
0 \\
0 \\
1 \\
0 \\
0 \\
0 \\
0 \\
0 \\
0 \\
0 \\
0 \\
0 \\
0 \\
0\end{array}$ & $\begin{array}{l}1 \\
1 \\
2 \\
1 \\
1 \\
1 \\
2 \\
6 \\
2 \\
3 \\
0 \\
3 \\
0 \\
2 \\
1 \\
4 \\
3 \\
1 \\
3 \\
1 \\
0 \\
1\end{array}$ & \begin{tabular}{l|l|}
3 & \\
0 & \\
0 & \\
0 \\
1 \\
2 \\
2 \\
0 \\
0 \\
0 \\
1 \\
1 \\
2 \\
1 \\
0 \\
1 \\
0 \\
1 \\
0 \\
1 \\
0 \\
0
\end{tabular} & \begin{tabular}{|l|}
0 \\
0 \\
0 \\
0 \\
0 \\
0 \\
0 \\
1 \\
0 \\
0 \\
1 \\
1 \\
0 \\
0 \\
0 \\
0 \\
0 \\
0 \\
0 \\
0 \\
0 \\
0
\end{tabular} & $\begin{array}{l}3 \\
0 \\
0 \\
4 \\
2 \\
0 \\
1 \\
2 \\
3 \\
0 \\
0 \\
1 \\
1 \\
2 \\
2 \\
2 \\
1 \\
0 \\
1 \\
2 \\
0 \\
0\end{array}$ & \begin{tabular}{l|l}
0 & \\
1 & \\
0 & \\
1 & \\
0 & \\
0 & \\
0 & \\
0 & \\
0 & \\
0 & \\
0 & \\
1 & \\
1 & \\
1 & \\
0 & \\
0 & \\
0 & \\
0 & \\
2 & \\
0 & \\
0 &
\end{tabular} & $\begin{array}{l}0 \\
0 \\
0 \\
0 \\
0 \\
0 \\
0 \\
1 \\
0 \\
0 \\
0 \\
0 \\
0 \\
0 \\
0 \\
0 \\
0 \\
0 \\
0 \\
0 \\
0 \\
0\end{array}$ & $\begin{array}{l}3 \\
0 \\
0 \\
0 \\
2 \\
0 \\
1 \\
1 \\
1 \\
2 \\
0 \\
1 \\
0 \\
1 \\
1 \\
0 \\
0 \\
0 \\
0 \\
1 \\
0 \\
0\end{array}$ & $\begin{array}{l}2 \\
0 \\
1 \\
0 \\
0 \\
0 \\
1 \\
1 \\
0 \\
0 \\
0 \\
0 \\
0 \\
0 \\
0 \\
0 \\
0 \\
0 \\
0 \\
0 \\
0 \\
0\end{array}$ & 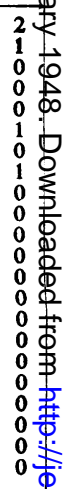 \\
\hline Transit & $\begin{array}{l}29 \\
30 \\
31 \\
32 \\
33\end{array}$ & \begin{tabular}{l|l}
0 \\
1 \\
2 \\
0 \\
0
\end{tabular} & \begin{tabular}{l|l}
0 & \\
1 & \\
0 & \\
0 & \\
0 &
\end{tabular} & $\begin{array}{l}0 \\
0 \\
0 \\
0 \\
0\end{array}$ & \begin{tabular}{l|l|l}
4 \\
0 \\
3 \\
0 \\
0
\end{tabular} & $\begin{array}{l}2 \\
0 \\
2 \\
1 \\
0\end{array}$ & $\begin{array}{l}2 \\
0 \\
0 \\
0 \\
0\end{array}$ & $\begin{array}{l}3 \\
0 \\
8 \\
1 \\
0\end{array}$ & $\begin{array}{l}0 \\
1 \\
0 \\
2 \\
0\end{array}$ & $\begin{array}{l}0 \\
0 \\
1 \\
0 \\
0\end{array}$ & $\begin{array}{l}3 \\
0 \\
2 \\
0 \\
0\end{array}$ & $\begin{array}{l}1 \\
0 \\
0 \\
1 \\
0\end{array}$ & \begin{tabular}{l|l}
1 & \\
0 & \\
0 & \\
0 & \\
0 &
\end{tabular} & $\begin{array}{l}3 \\
1 \\
3 \\
0 \\
0\end{array}$ & $\begin{array}{l}0 \\
0 \\
1 \\
0 \\
0\end{array}$ & $\begin{array}{l}0 \\
0 \\
0 \\
0 \\
0\end{array}$ & $\begin{array}{l}1 \\
1 \\
1 \\
0 \\
0\end{array}$ & \begin{tabular}{l|}
0 \\
0 \\
2 \\
0 \\
0
\end{tabular} & $\begin{array}{l}0 \\
0 \\
0 \\
0 \\
0\end{array}$ & $\begin{array}{l}6 \\
0 \\
0 \\
0 \\
0\end{array}$ & \begin{tabular}{l|l}
2 & \\
1 & \\
0 & \\
0 & \\
0 &
\end{tabular} & $\begin{array}{l}0 \\
0 \\
0 \\
0 \\
0\end{array}$ & $\begin{array}{l}0 \\
3 \\
1 \\
0 \\
0\end{array}$ & \begin{tabular}{l|l}
0 & \\
1 \\
1 \\
0 \\
0
\end{tabular} & $\begin{array}{l}0 \text { 电 } \\
0 \\
0 \\
0 \\
0 \\
0\end{array}$ \\
\hline $\begin{array}{l}\text { Total } \\
\text { ity (cla } \\
\text { Total } \\
\text { rate p } \\
\text { (classi }\end{array}$ & $\begin{array}{l}\text { morbid- } \\
\text { assified) } \\
\text { morbid- } \\
\text { er } 1,000 \\
\text { fied) }\end{array}$ & $\begin{array}{r}126 \\
567 \cdot 0\end{array}$ & $\begin{array}{l}42 \\
298 \cdot 25\end{array}$ & $52 \cdot 24$ & $\begin{array}{l}111 \\
499 \cdot 52\end{array}$ & $\begin{array}{l}42 \\
298 \cdot 25\end{array}$ & $52 \cdot 24$ & $\begin{array}{r}107 \\
481 \cdot 5\end{array}$ & $227 \cdot 2$ & $\begin{array}{c}13 \\
75 \cdot 42\end{array}$ & $\begin{array}{c}60 \\
270 \cdot 0\end{array}$ & 184.6 & $\begin{array}{l}11 \\
63 \cdot 8 \cdot 2\end{array}$ & $\begin{array}{l}57 \\
256 \cdot 5: 1\end{array}$ & $\begin{array}{r}19 \\
134.917\end{array}$ & $\begin{array}{c}3 \\
17 \cdot 4\end{array}$ & $198 \cdot 0$ & $\mid$\begin{tabular}{|c|}
20 \\
$142 \cdot 0$
\end{tabular} & $\begin{array}{c}5 \\
29 \cdot 0\end{array}$ & $\begin{array}{c}41 \\
184 \cdot 5\end{array}$ & $\begin{array}{c}14 \\
99.4\end{array}$ & $\begin{array}{c:c}1 & \\
5 \cdot 8 & 1\end{array}$ & $\begin{array}{c}29 \\
130 \cdot 5\end{array}$ & $\begin{array}{c}10 \\
71 \cdot 0\end{array}$ & $\begin{array}{r}8 \frac{8}{0} \\
46.98\end{array}$ \\
\hline $\begin{array}{c}\text { Total } \\
\text { ity }\end{array}$ & morbid- & & 177 & & & 162 & & & 152 & & & 97 & & & 79 & & & 69 & & & 56 & & & 47 & \\
\hline $\begin{array}{l}\text { Total r } \\
\text { ity ra } \\
1,000\end{array}$ & $\begin{array}{l}\text { morbid- } \\
\text { ate per }\end{array}$ & & $29 \cdot 2$ & & & $01 \cdot 3$ & & & $282 \cdot 7$ & & & $180 \cdot 4$ & & & 146.9 & & & $128.3^{\circ}$ & & & $104 \cdot 2$ & & & $87 \cdot 4$ & \\
\hline & & & & & & & & & & & & & & & & & & & & & & & & & 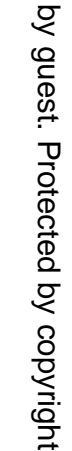 \\
\hline
\end{tabular}


WeEk for the Three Occupational Groups

\begin{tabular}{|c|c|c|c|c|c|c|c|c|c|c|c|c|c|c|c|c|c|c|c|c|c|c|c|c|c|}
\hline \multicolumn{3}{|c|}{$\begin{array}{c}\text { Diseases of } \\
\text { upper } \\
\text { respiratory } \\
\text { tract } \\
9\end{array}$} & \multicolumn{3}{|c|}{$\begin{array}{c}\text { Burns and } \\
\text { scalds } \\
10\end{array}$} & \multicolumn{3}{|c|}{$\begin{array}{c}\begin{array}{c}\text { Dental } \\
\text { conditions }\end{array} \\
11\end{array}$} & \multicolumn{3}{|c|}{$\begin{array}{c}\text { Disorders } \\
\text { of C.N.S. } \\
12\end{array}$} & \multicolumn{3}{|c|}{$\begin{array}{c}\text { Diarrboea } \\
13\end{array}$} & \multicolumn{3}{|c|}{$\begin{array}{c}\text { Miscellaneous } \\
\text { conditions } \\
14\end{array}$} & \multicolumn{3}{|c|}{ Weokly morbidity } & \multicolumn{3}{|c|}{$\begin{array}{c}\text { Weekly } \\
\text { morbidity } \\
\text { rate } \\
\text { per } 1,000\end{array}$} & \multirow[t]{2}{*}{$\begin{array}{c}\text { Total } \\
\text { weekly } \\
\text { morbid- } \\
\text { ity }\end{array}$} & \multirow[t]{2}{*}{$\begin{array}{c}\text { Total } \\
\text { weekly } \\
\text { morbid- } \\
\text { ity rate } \\
\text { per } 1,000\end{array}$} \\
\hline $\mathbf{A}$ & B & C & $\mathbf{A}$ & B & C & $\mathbf{A}$ & B & C & $\mathbf{A}$ & B & C & $\bar{A}$ & B & $\bar{C}$ & $\mathbf{A}$ & B & $\mathbf{C}$ & $\bar{A}$ & B & C & $\mathbf{A}$ & B & $\mathbf{C}$ & & \\
\hline \begin{tabular}{l|}
0 \\
1 \\
1 \\
2 \\
1 \\
0
\end{tabular} & $\begin{array}{l}0 \\
0 \\
1 \\
1 \\
0 \\
0\end{array}$ & $\begin{array}{l}0 \\
0 \\
0 \\
1 \\
0 \\
1\end{array}$ & $\begin{array}{l}0 \\
1 \\
0 \\
0 \\
1 \\
1\end{array}$ & \begin{tabular}{l|l}
0 \\
0 \\
1 \\
0 \\
1 \\
0
\end{tabular} & $\begin{array}{l}0 \\
0 \\
0 \\
0 \\
0 \\
0\end{array}$ & $\begin{array}{l}0 \\
1 \\
0 \\
0 \\
0 \\
1\end{array}$ & $\begin{array}{l}\mathbf{0} \\
\mathbf{0} \\
\mathbf{0} \\
\mathbf{0} \\
\mathbf{0} \\
\mathbf{0}\end{array}$ & $\begin{array}{l}0 \\
0 \\
0 \\
0 \\
1 \\
0\end{array}$ & $\begin{array}{l}0 \\
0 \\
0 \\
0 \\
0 \\
0\end{array}$ & $\begin{array}{l}0 \\
0 \\
0 \\
0 \\
0 \\
0\end{array}$ & $\begin{array}{l}0 \\
0 \\
1 \\
0 \\
0 \\
0\end{array}$ & $\begin{array}{l}0 \\
0 \\
1 \\
1 \\
0 \\
0\end{array}$ & $\begin{array}{l}0 \\
0 \\
0 \\
0 \\
0 \\
0\end{array}$ & $\begin{array}{l}0 \\
0 \\
0 \\
1 \\
0 \\
1\end{array}$ & $\begin{array}{l}0 \\
0 \\
0 \\
0 \\
0 \\
1\end{array}$ & $\begin{array}{l}1 \\
0 \\
0 \\
3 \\
0 \\
0\end{array}$ & $\begin{array}{l}1 \\
0 \\
0 \\
0 \\
0 \\
0\end{array}$ & $\begin{array}{r}1 \\
7 \\
10 \\
21 \\
19 \\
13\end{array}$ & $\begin{array}{r}3 \\
1 \\
12 \\
13 \\
14 \\
8\end{array}$ & $\begin{array}{l}2 \\
1 \\
3 \\
7 \\
5 \\
3\end{array}$ & \begin{tabular}{|c|}
4.5 \\
31.5 \\
45.0 \\
94.5 \\
85.5 \\
58.5
\end{tabular} & \begin{tabular}{|c|}
21.3 \\
7.1 \\
85.2 \\
92.3 \\
99.4 \\
56.8
\end{tabular} & \begin{tabular}{|l|}
11.6 \\
5.8 \\
$17 \cdot 4$ \\
$40 \cdot 6$ \\
$29 \cdot 0$ \\
17.4
\end{tabular} & $\begin{array}{r}6 \\
95 \\
25 \\
41 \\
38 \\
24\end{array}$ & $\begin{array}{l}11 \cdot 2 \\
16 \cdot 7 \\
46 \cdot 5 \\
76 \cdot 3 \\
70 \cdot 7 \\
44 \cdot 6\end{array}$ \\
\hline $\begin{array}{l}1 \\
2 \\
1 \\
1 \\
0 \\
0 \\
0 \\
0 \\
1 \\
0 \\
0 \\
1 \\
0 \\
3 \\
0 \\
1 \\
1 \\
3 \\
0 \\
0 \\
0 \\
0 \\
\end{array}$ & $\begin{array}{l}2 \\
0 \\
1 \\
1 \\
2 \\
0 \\
0 \\
1 \\
0 \\
0 \\
0 \\
0 \\
1 \\
0 \\
1 \\
0 \\
0 \\
1 \\
0 \\
0 \\
0 \\
0 \\
\end{array}$ & $\begin{array}{l}0 \\
0 \\
0 \\
0 \\
0 \\
0 \\
0 \\
0 \\
0 \\
0 \\
0 \\
0 \\
0 \\
0 \\
0 \\
0 \\
0 \\
0 \\
0 \\
0 \\
0 \\
0\end{array}$ & $\begin{array}{l}1 \\
0 \\
0 \\
1 \\
1 \\
0 \\
0 \\
2 \\
0 \\
1 \\
0 \\
0 \\
0 \\
1 \\
1 \\
0 \\
0 \\
1 \\
0 \\
0 \\
\end{array}$ & $\begin{array}{l}2 \\
1 \\
0 \\
0 \\
1 \\
0 \\
0 \\
1 \\
2 \\
0 \\
0 \\
0 \\
0 \\
1 \\
0 \\
0 \\
0 \\
0 \\
1 \\
0 \\
0 \\
1 \\
\end{array}$ & $\begin{array}{l}0 \\
0 \\
0 \\
0 \\
0 \\
0 \\
1 \\
0 \\
0 \\
0 \\
0 \\
0 \\
0 \\
0 \\
1 \\
0 \\
0 \\
0 \\
0 \\
0 \\
0 \\
0\end{array}$ & $\begin{array}{l}0 \\
1 \\
0 \\
0 \\
3 \\
0 \\
0 \\
2 \\
1 \\
2 \\
2 \\
1 \\
1 \\
2 \\
0 \\
1 \\
0 \\
0 \\
1 \\
1 \\
2 \\
0 \\
\end{array}$ & $\begin{array}{l}1 \\
0 \\
0 \\
0 \\
0 \\
0 \\
0 \\
0 \\
1 \\
1 \\
0 \\
0 \\
0 \\
0 \\
0 \\
0 \\
0 \\
0 \\
0 \\
1 \\
0 \\
2 \\
\end{array}$ & $\begin{array}{l}0 \\
0 \\
0 \\
0 \\
0 \\
0 \\
0 \\
0 \\
0 \\
0 \\
0 \\
1 \\
0 \\
0 \\
0 \\
0 \\
0 \\
0 \\
0 \\
0 \\
0 \\
0\end{array}$ & $\begin{array}{l}0 \\
0 \\
0 \\
2 \\
0 \\
0 \\
0 \\
2 \\
0 \\
0 \\
0 \\
0 \\
0 \\
1 \\
0 \\
0 \\
0 \\
2 \\
2 \\
0 \\
1\end{array}$ & \begin{tabular}{|l|}
0 \\
0 \\
0 \\
0 \\
0 \\
0 \\
0 \\
1 \\
1 \\
0 \\
1 \\
0 \\
0 \\
0 \\
0 \\
1 \\
0 \\
0 \\
0 \\
1 \\
1 \\
0 \\
\end{tabular} & $\begin{array}{l}0 \\
0 \\
0 \\
0 \\
0 \\
0 \\
0 \\
0 \\
0 \\
0 \\
0 \\
0 \\
1 \\
0 \\
1 \\
0 \\
1 \\
0 \\
0 \\
0 \\
0\end{array}$ & $\begin{array}{l}1 \\
0 \\
0 \\
0 \\
0 \\
0 \\
0 \\
1 \\
0 \\
0 \\
1 \\
0 \\
0 \\
0 \\
0 \\
0 \\
0 \\
0 \\
2 \\
0 \\
0 \\
0\end{array}$ & $\begin{array}{l}1 \\
0 \\
0 \\
0 \\
0 \\
0 \\
1 \\
0 \\
0 \\
0 \\
0 \\
0 \\
0 \\
0 \\
0 \\
0 \\
0 \\
0 \\
0 \\
0 \\
0\end{array}$ & $\begin{array}{l}0 \\
0 \\
0 \\
0 \\
0 \\
0 \\
0 \\
0 \\
0 \\
0 \\
0 \\
0 \\
0 \\
0 \\
0 \\
0 \\
0 \\
0 \\
0 \\
0 \\
0 \\
0\end{array}$ & $\begin{array}{l}2 \\
0 \\
1 \\
1 \\
3 \\
2 \\
2 \\
0 \\
1 \\
3 \\
0 \\
1 \\
0 \\
2 \\
0 \\
1 \\
0 \\
2 \\
2 \\
1 \\
0 \\
0\end{array}$ & $\begin{array}{l}1 \\
1 \\
0 \\
0 \\
1 \\
6 \\
0 \\
0 \\
0 \\
0 \\
0 \\
0 \\
1 \\
1 \\
0 \\
1 \\
1 \\
0 \\
1 \\
0 \\
0 \\
1\end{array}$ & $\begin{array}{l}0 \\
1 \\
0 \\
1 \\
0 \\
0 \\
0 \\
0 \\
0 \\
0 \\
0 \\
0 \\
0 \\
0 \\
0 \\
1 \\
0 \\
0 \\
0 \\
0 \\
0 \\
0\end{array}$ & $\begin{array}{c}27 \\
23 \\
19 \\
40 \\
48 \\
24 \\
21 \\
39 \\
15 \\
29 \\
-19 \\
28 \\
17 \\
30 \\
16 \\
15 \\
20 \\
27 \\
29 \\
31 \\
20 \\
17\end{array}$ & $\begin{array}{r}24 \\
8 \\
4 \\
9 \\
12 \\
4 \\
7 \\
15 \\
6 \\
9 \\
5 \\
6 \\
7 \\
14 \\
2 \\
9 \\
7 \\
6 \\
13 \\
8 \\
4 \\
7\end{array}$ & $\begin{array}{l}4 \\
5 \\
1 \\
4 \\
1 \\
4 \\
3 \\
4 \\
3 \\
0 \\
4 \\
2 \\
4 \\
1 \\
2 \\
1 \\
1 \\
0 \\
0 \\
0 \\
4 \\
2\end{array}$ & \begin{tabular}{|c|}
$121 \cdot 5$ \\
$103 \cdot 5$ \\
85.5 \\
$180 \cdot 0$ \\
216.0 \\
168.0 \\
$94 \cdot 5$ \\
175.5 \\
$67 \cdot 5$ \\
130.5 \\
85.5 \\
126.0 \\
76.5 \\
135.0 \\
72.0 \\
67.5 \\
$90 \cdot 0$ \\
121.5 \\
130.5 \\
$139 \cdot 5$ \\
990.0 \\
76.5
\end{tabular} & \begin{tabular}{|l|}
70.4 \\
56.8 \\
28.4 \\
63.9 \\
85.2 \\
28.4 \\
49.7 \\
106.5 \\
42.6 \\
63.9 \\
35.5 \\
42.6 \\
49.7 \\
99.4 \\
14.2 \\
63.9 \\
49.7 \\
42.6 \\
92.3 \\
56.8 \\
28.4 \\
49.7
\end{tabular} & \begin{tabular}{r|}
$23 \cdot 2$ \\
$29 \cdot 0$ \\
$5 \cdot 8$ \\
$23 \cdot 2$ \\
$5 \cdot 8$ \\
$23 \cdot 2$ \\
$17 \cdot 4$ \\
$23 \cdot 2$ \\
$17 \cdot 4$ \\
0.0 \\
$23 \cdot 2$ \\
$11 \cdot 6$ \\
$23 \cdot 2$ \\
$5 \cdot 8$ \\
$11 \cdot 6$ \\
$5 \cdot 8$ \\
$5 \cdot 8$ \\
0.0 \\
0.0 \\
0.0 \\
$23 \cdot 2$ \\
$11 \cdot 6$
\end{tabular} & $\begin{array}{l}55 \\
36 \\
24 \\
53 \\
61 \\
32 \\
31 \\
58 \\
24 \\
38 \\
28 \\
36 \\
28 \\
45 \\
20 \\
25 \\
28 \\
33 \\
42 \\
39 \\
28 \\
26\end{array}$ & $\begin{array}{r}102.3 \\
67.0 \\
44.6 \\
98.6 \\
113.5 \\
59.5 \\
57.7 \\
107.9 \\
44.6 \\
70.7 \\
52.1 \\
67.0 \\
52.1 \\
83.7 \\
37.2 \\
46.5 \\
52.1 \\
61.4 \\
78.1 \\
72.5 \\
52.1 \\
48.4\end{array}$ \\
\hline $\begin{array}{l}1 \\
0 \\
1 \\
0 \\
0\end{array}$ & $\begin{array}{l}0 \\
1 \\
0 \\
0 \\
0\end{array}$ & $\begin{array}{l}\mathbf{0} \\
\mathbf{0} \\
\mathbf{0} \\
\mathbf{0}\end{array}$ & $\begin{array}{l}4 \\
0 \\
0 \\
0 \\
0\end{array}$ & $\begin{array}{l}1 \\
0 \\
1 \\
0 \\
0\end{array}$ & $\begin{array}{l}0 \\
0 \\
0 \\
0 \\
0\end{array}$ & $\begin{array}{l}1 \\
0 \\
0 \\
0 \\
0\end{array}$ & $\begin{array}{l}0 \\
0 \\
0 \\
0 \\
0\end{array}$ & $\begin{array}{l}\mathbf{0} \\
\mathbf{0} \\
\mathbf{0} \\
\mathbf{0} \\
\mathbf{0}\end{array}$ & $\begin{array}{l}0 \\
0 \\
1 \\
1 \\
0\end{array}$ & $\begin{array}{l}0 \\
0 \\
1 \\
0 \\
0\end{array}$ & $\begin{array}{l}0 \\
0 \\
0 \\
0 \\
0\end{array}$ & \begin{tabular}{l|}
0 \\
1 \\
0 \\
1 \\
0
\end{tabular} & $\begin{array}{l}0 \\
0 \\
0 \\
0 \\
0\end{array}$ & $\begin{array}{l}0 \\
1 \\
0 \\
0 \\
0\end{array}$ & $\begin{array}{l}0 \\
0 \\
2 \\
1 \\
0\end{array}$ & $\begin{array}{r}2 \\
\cdot 1 \\
1 \\
0 \\
0\end{array}$ & $\begin{array}{l}0 \\
0 \\
0 \\
0 \\
0\end{array}$ & $\begin{array}{r}26 \\
7 \\
24 \\
4 \\
0\end{array}$ & $\begin{array}{l}8 \\
6 \\
9 \\
4 \\
0\end{array}$ & $\begin{array}{l}3 \\
1 \\
1 \\
0 \\
0\end{array}$ & \begin{tabular}{|r|}
117.0 \\
31.5 \\
108.0 \\
18.0 \\
0.0
\end{tabular} & \begin{tabular}{|r|}
56.8 \\
42.6 \\
63.9 \\
28.4 \\
0.0
\end{tabular} & \begin{tabular}{r|}
17.4 \\
5.8 \\
5.8 \\
0.0 \\
0.0
\end{tabular} & $\begin{array}{r}37 \\
14 \\
34 \\
8 \\
0\end{array}$ & $\begin{array}{r}68.8 \\
26.0 \\
63.2 \\
14.9 \\
0.0\end{array}$ \\
\hline 22 & 13 & 2 & 17 & 14 & 2 & 23 & 6 & 2 & 12 & 7 & 4 & 9 & 2 & 3 & 28 & 17 & 4 & & 264 & 76 & & & & 1,026 & \\
\hline & 37 & & & 33 & & & 31 & & & 23 & & & 14 & & & 49 & & & 1,026 & & & & & 1,026 & \\
\hline & 68.8 & & & $61 \cdot 4$ & & & $57 \cdot 7$ & & & $42 \cdot 8$ & & & $26 \cdot 0$ & & & $91 \cdot 1$ & & & $1908 \cdot 4$ & & & & & & \\
\hline
\end{tabular}

$\stackrel{0}{\overrightarrow{1}}$

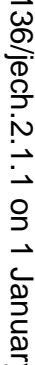

$\stackrel{\vec{\phi}}{\infty}$

$\square$

之o

흥

\%

$\stackrel{8}{\circ}$

혹

롱

त) 


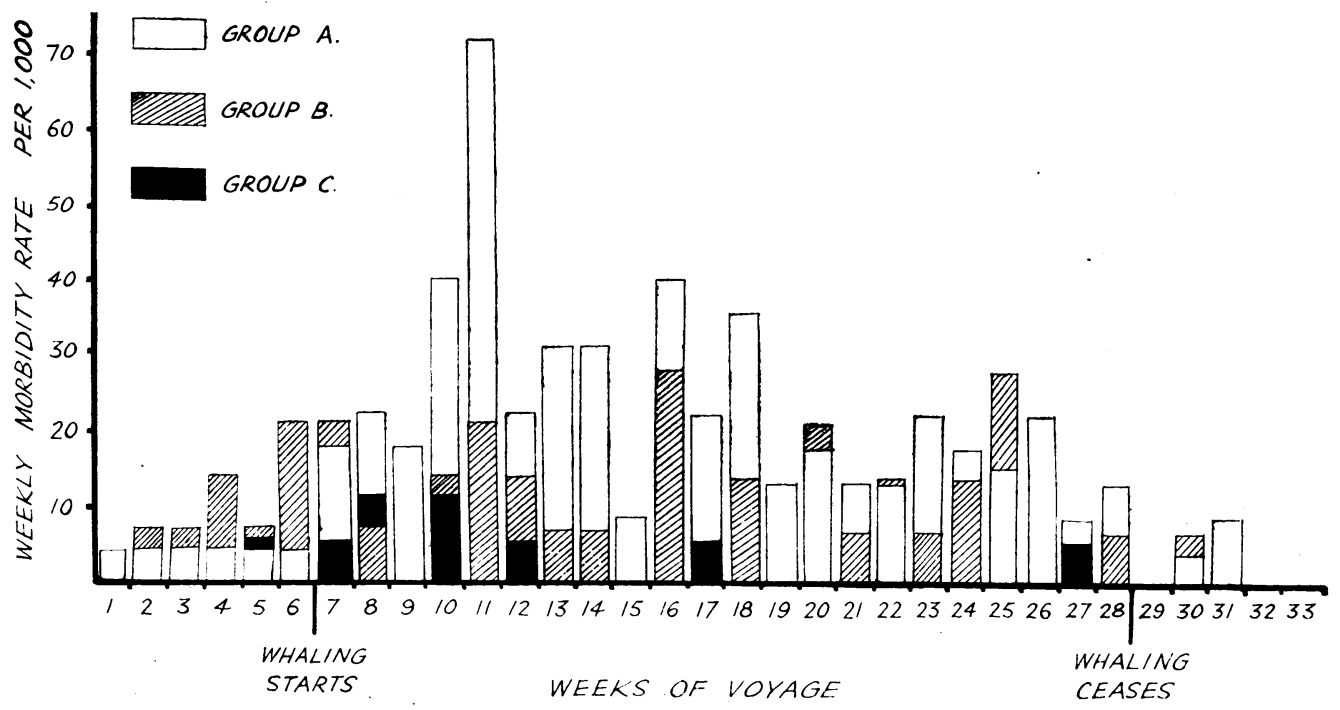

FIG. 1.-Weekly morbidity rate per 1,000 for each group for Column 1 (suppurative lesions).

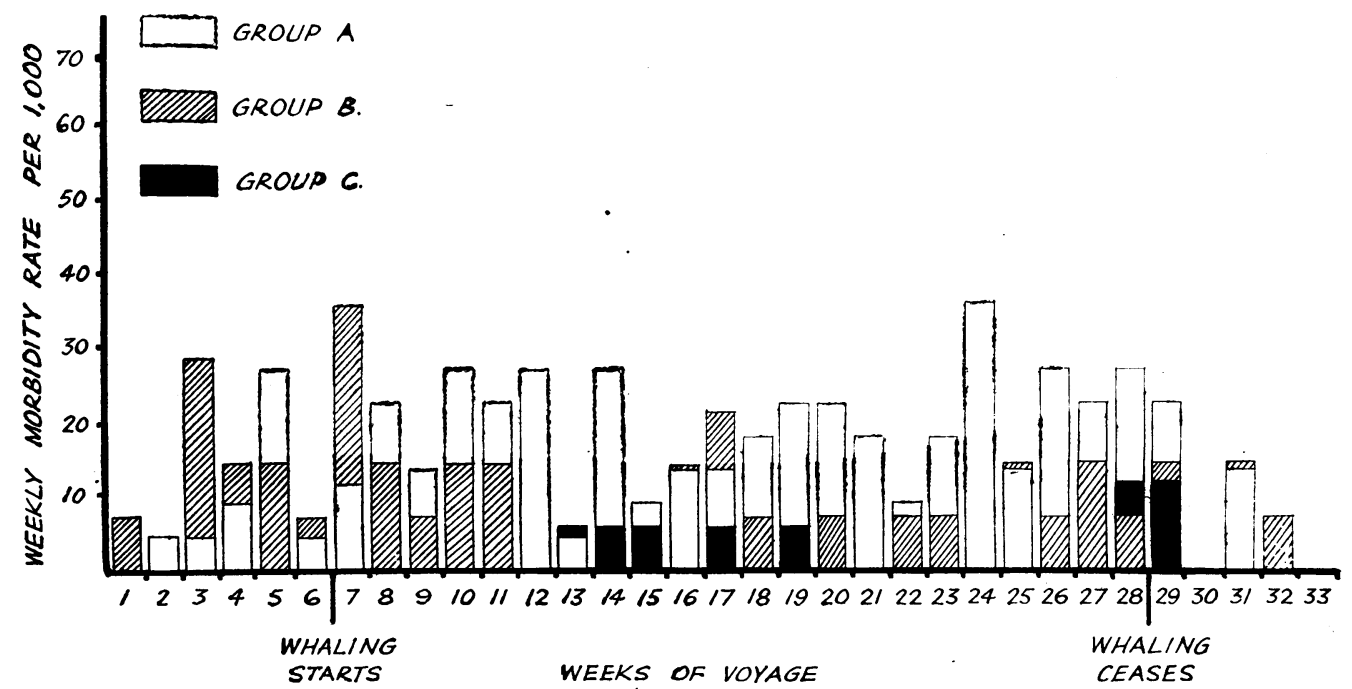

FIG. 2.-Weekly morbidity rate per 1,000 for each group for Column 2 (traumatic conditions including fractures, without sepsis). 


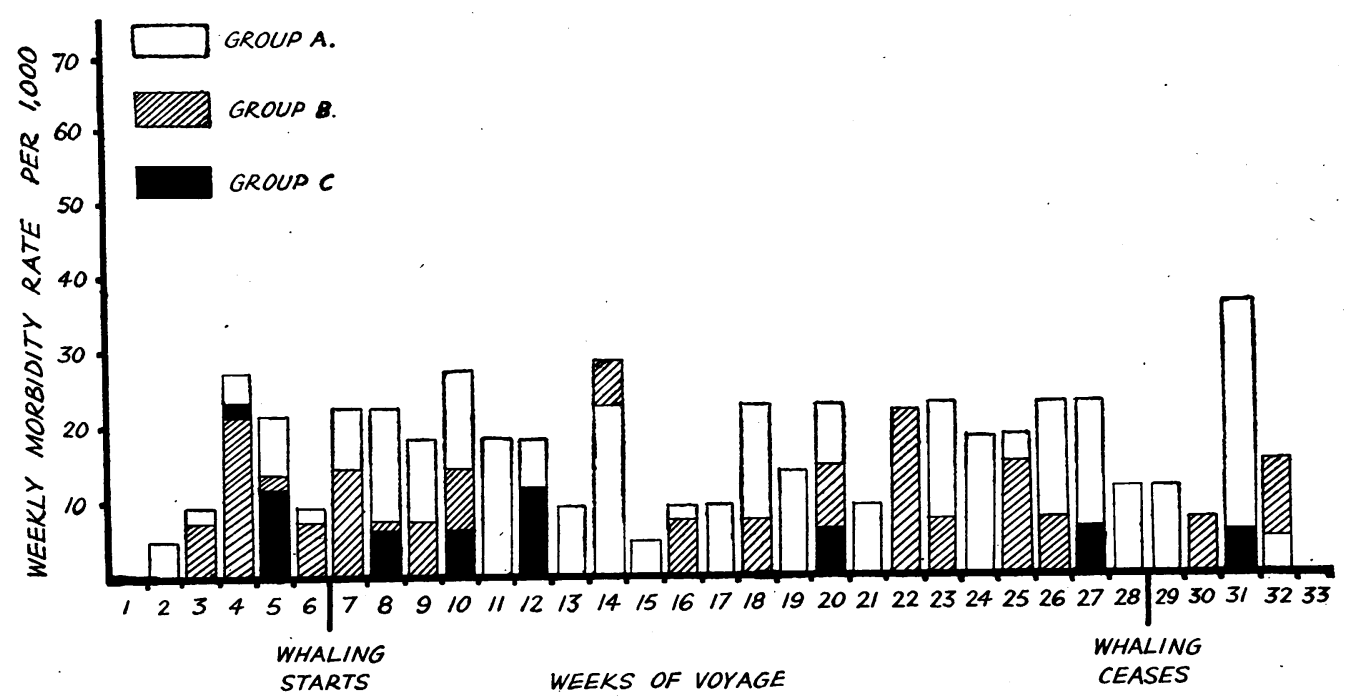

Fig. 3.-Weekly morbidity rate per 1,000 for each group for Column 3 (diseases of the skin).

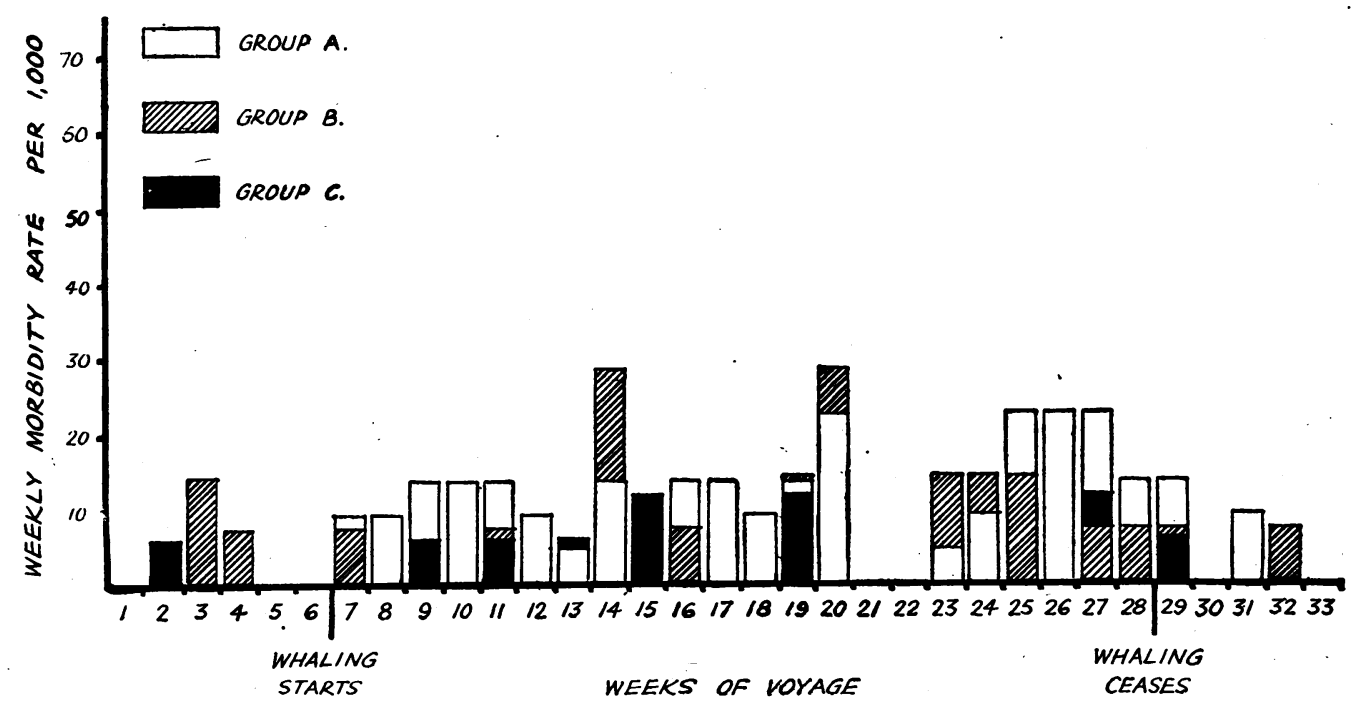

FIG. 4.-Weekly morbidity rate per 1,000 for each group for Column 4 (alimentary disorders other than diarrhoea). 


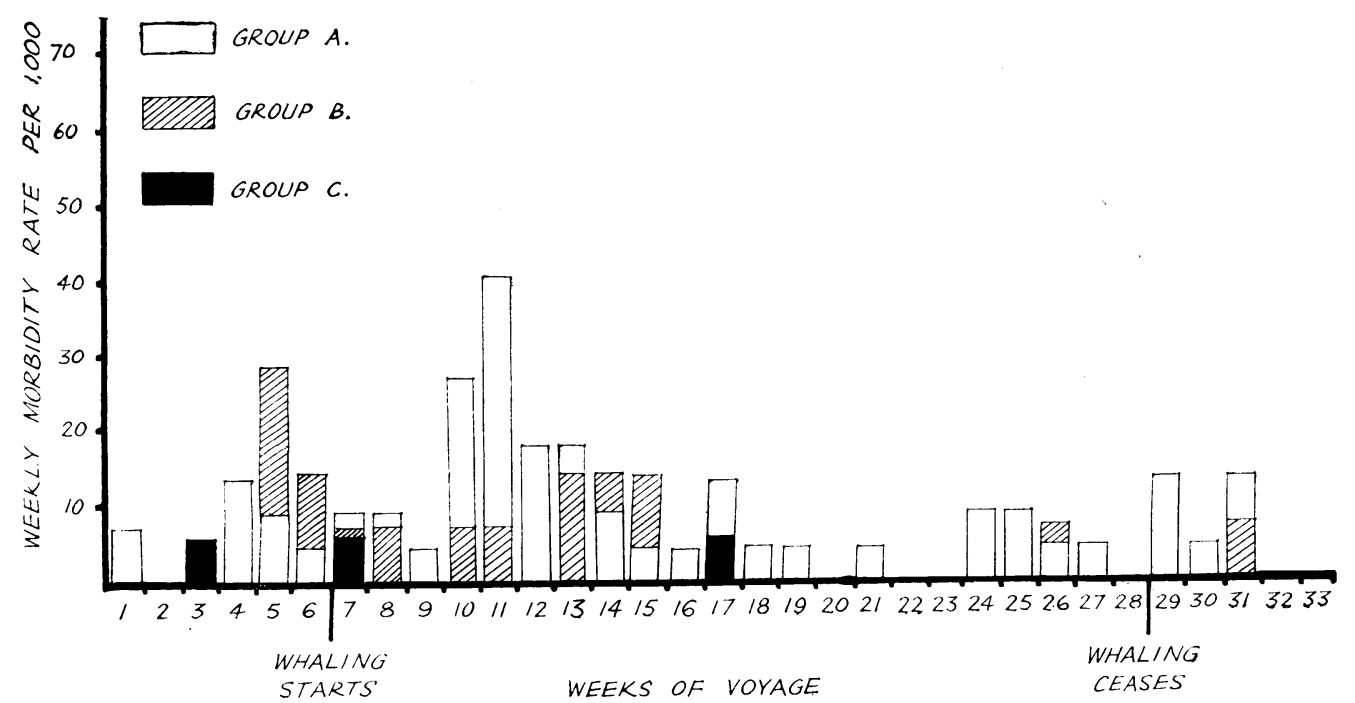

FIG. 5.-Weekly morbidity rate per 1,000 for each group for Column 5 (diseases of the ear, nose, and throat).

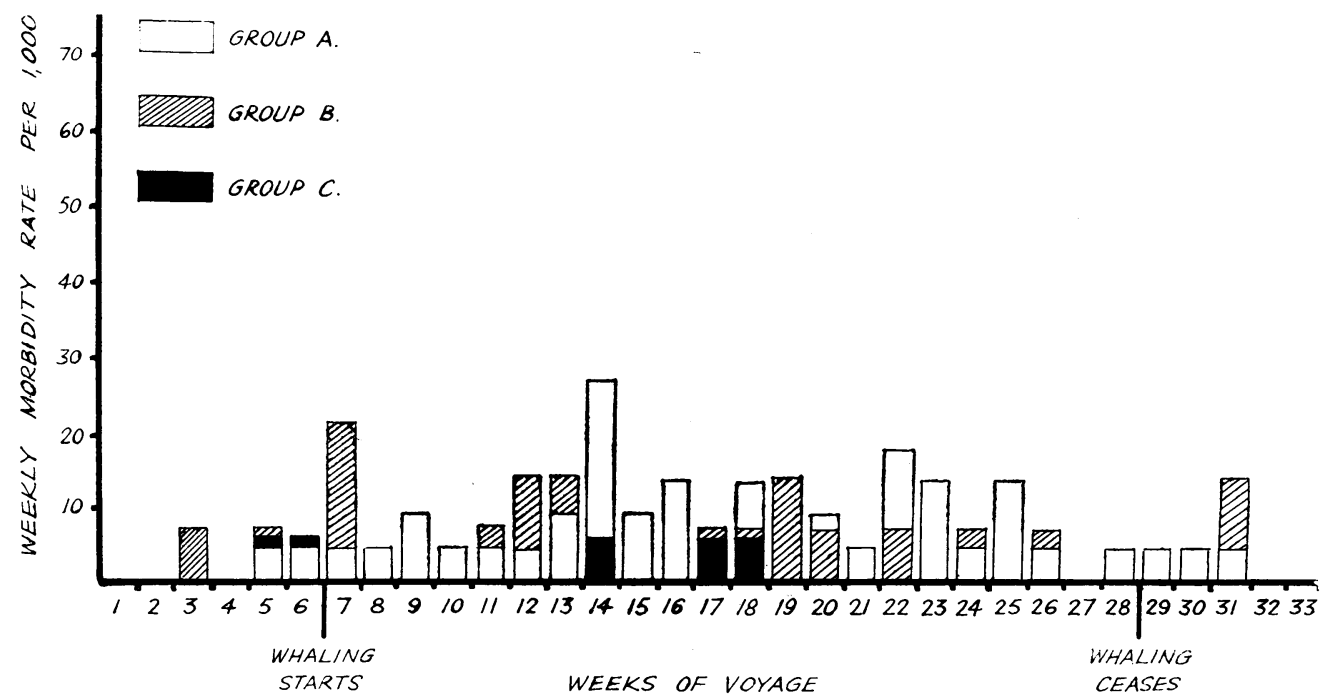

Fig. 6.-Weekly morbidity rate per 1,000 for each group for Column 6 (" rheumatic " conditions). 


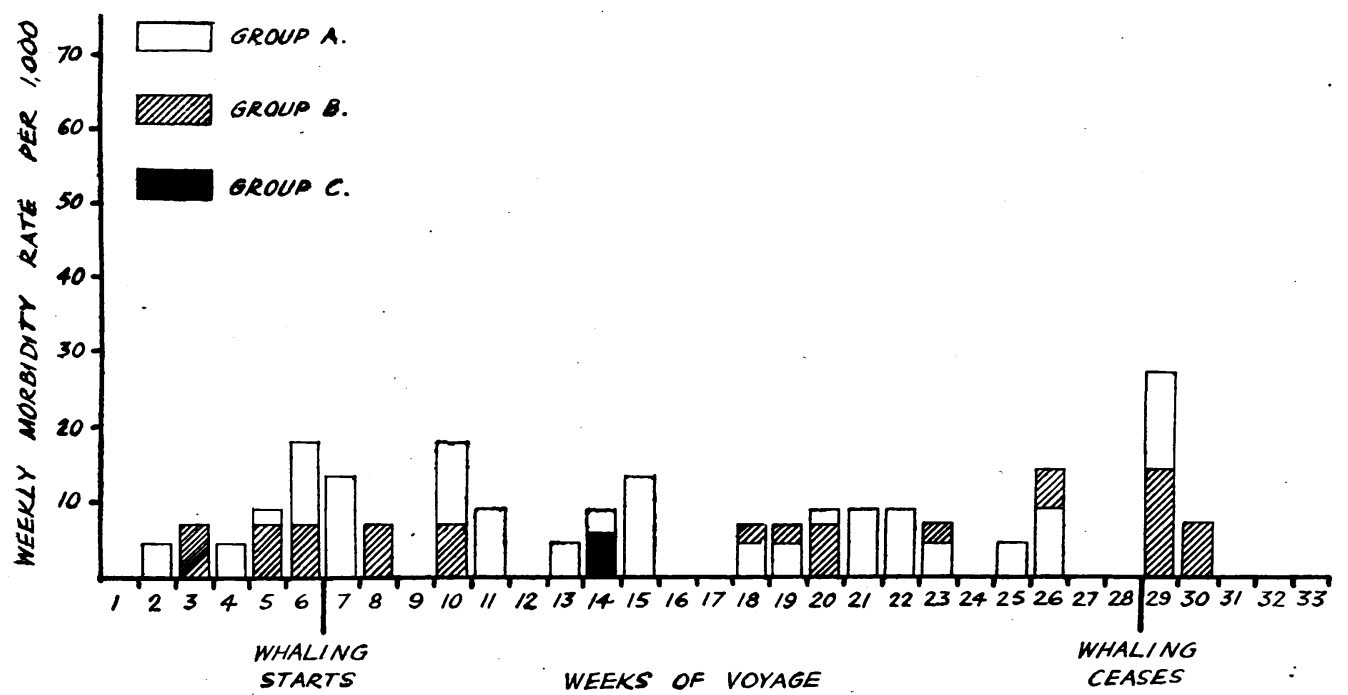

FIO. 7.-Weekly morbidity rate per 1,000 for each group for Column 7 (diseases including foreign bodies of the eye).

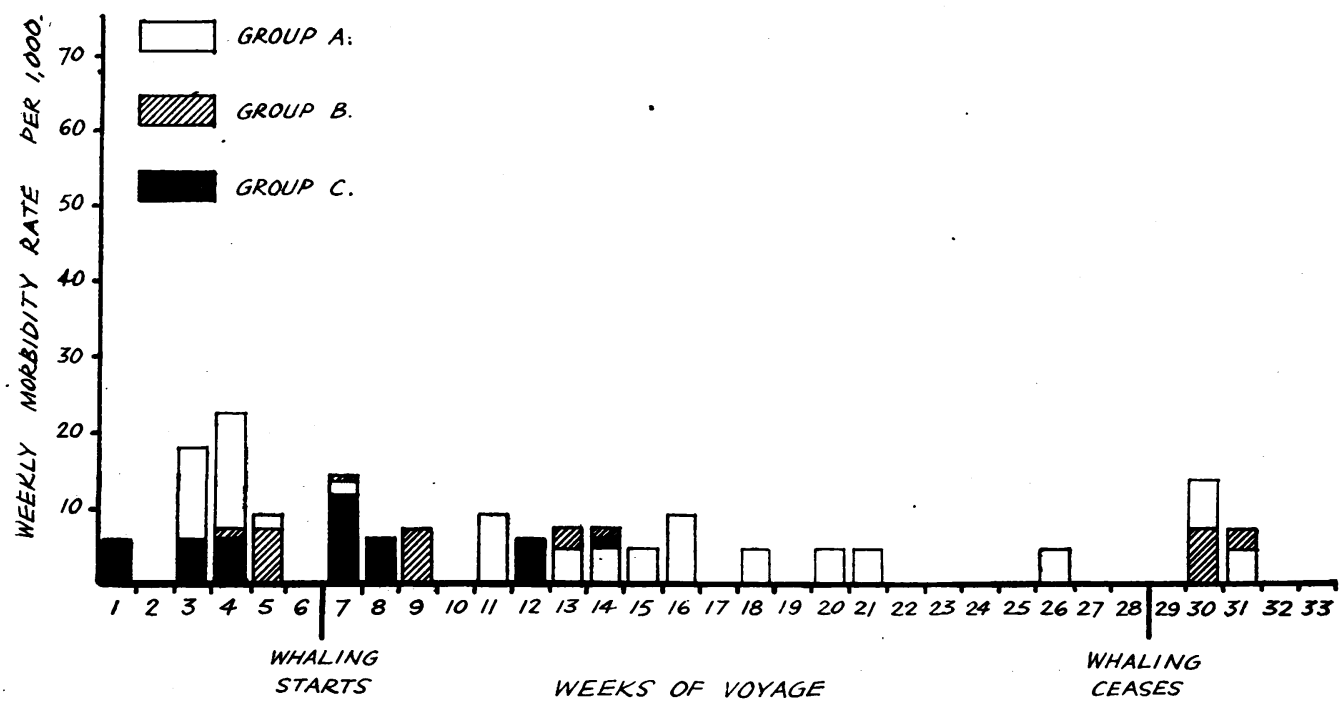

Fig. 8. Weekly morbidity rate per 1,000 for each group for Column 8 (venereal diseases, all types). 
TABLE

WEEKLY MORBIDITY RATE PER

\begin{tabular}{|c|c|c|c|c|c|c|c|c|c|c|c|c|c|c|c|c|c|c|c|c|c|c|c|}
\hline Period & $\begin{array}{c}\text { Week } \\
\text { of } \\
\text { voyage }\end{array}$ & \multicolumn{3}{|c|}{$\begin{array}{c}\begin{array}{c}\text { Suppurative } \\
\text { lesions }\end{array} \\
1\end{array}$} & \multicolumn{3}{|c|}{$\begin{array}{c}\begin{array}{c}\text { Trauma and } \\
\text { fractures } \\
\text { without sepsis }\end{array} \\
2\end{array}$} & \multicolumn{3}{|c|}{$\begin{array}{c}\text { Diseases of } \\
\text { the skin } \\
3\end{array}$} & \multicolumn{3}{|c|}{$\begin{array}{c}\text { Non-diarrhoeic } \\
\text { alimentary } \\
\text { disorders } \\
4\end{array}$} & \multicolumn{3}{|c|}{$\begin{array}{c}\text { Diseases of } \\
\text { ear, nose, and } \\
\text { throat } \\
5\end{array}$} & \multicolumn{3}{|c|}{$\begin{array}{c}\text { "Rheumatic " } \\
\text { conditions } \\
6\end{array}$} & \multicolumn{4}{|c|}{$\begin{array}{c}\text { Diseases of } \\
\text { the eye } \\
7\end{array}$} \\
\hline & roup & A & B & C & A & B & C & A & B & C & A & B & C & A & B & C & A & B & C & A & B & $\mathrm{C}$ & \\
\hline ransit & $\begin{array}{l}1 \\
2 \\
3 \\
4 \\
5 \\
6\end{array}$ & $\begin{array}{l}4 \cdot 5 \\
4.5 \\
4 \cdot 5 \\
4.5 \\
4 \cdot 5 \\
4 \cdot 5\end{array}$ & \begin{tabular}{r|}
$0 \cdot 0$ \\
$7 \cdot 1$ \\
$7 \cdot 1$ \\
$14 \cdot 2$ \\
7.1 \\
$21 \cdot 3$
\end{tabular} & $\begin{array}{l}0 \cdot 0 \\
0 \cdot 0 \\
0 \cdot 0 \\
0.0 \\
5 \cdot 8 \\
0.0\end{array}$ & \begin{tabular}{r|}
0.0 \\
4.5 \\
4.5 \\
9.0 \\
27.0 \\
4.5
\end{tabular} & \begin{tabular}{r|}
$7 \cdot 1$ \\
0.0 \\
$28 \cdot 4$ \\
$14 \cdot 2$ \\
$14 \cdot 2$ \\
$7 \cdot 1$
\end{tabular} & $\begin{array}{l}0 \cdot 0 \\
0 \cdot 0 \\
0 \cdot 0 \\
0 \cdot 0 \\
0 \cdot 0 \\
0.0\end{array}$ & \begin{tabular}{r|}
$0 \cdot 0$ \\
$4 \cdot 5$ \\
$9 \cdot 0$ \\
$27 \cdot 0$ \\
$13 \cdot 5$ \\
$9 \cdot 0$
\end{tabular} & \begin{tabular}{r|}
$0 \cdot 0$ \\
$0 \cdot 0$ \\
$7 \cdot 1$ \\
$21 \cdot 3$ \\
$21 \cdot 3$ \\
$7 \cdot 1$
\end{tabular} & \begin{tabular}{|c|}
$0 \cdot 0$ \\
$0 \cdot 0$ \\
$0 \cdot 0$ \\
$23 \cdot 2$ \\
$11 \cdot 6$ \\
$0 \cdot 0$
\end{tabular} & $\begin{array}{l}0.0 \\
0.0 \\
0.0 \\
0.0 \\
0.0 \\
0.0\end{array}$ & \begin{tabular}{r|}
0.0 \\
$0 \cdot 0$ \\
$14 \cdot 2$ \\
$7 \cdot 1$ \\
$0 \cdot 0$ \\
0.0
\end{tabular} & $\begin{array}{l}0.0 \\
5.8 \\
0 \cdot 0 \\
0 \cdot 0 \\
0.0 \\
0.0\end{array}$ & \begin{tabular}{r|}
0.0 \\
0.0 \\
0.0 \\
13.5 \\
9.0 \\
4.5
\end{tabular} & \begin{tabular}{r|}
$7 \cdot 1$ \\
$0 \cdot 0$ \\
$0 \cdot 0$ \\
0.0 \\
$28 \cdot 4$ \\
$14 \cdot 2$
\end{tabular} & & $\begin{array}{l}0 \cdot 0 \\
0 \cdot 0 \\
0 \cdot 0 \\
0 \cdot 0 \\
4 \cdot 5 \\
4 \cdot 5\end{array}$ & $\begin{array}{l}0.0 \\
0.0 \\
7.1 \\
0.0 \\
7.1 \\
0.0\end{array}$ & & \begin{tabular}{|r|}
0.0 \\
4.5 \\
0.0 \\
4.5 \\
9.0 \\
18.0
\end{tabular} & & $\begin{array}{l}0 \\
0\end{array}$ & \\
\hline Whalir & $\begin{array}{r}77 \\
8 \\
9 \\
10 \\
11 \\
12 \\
13 \\
14 \\
15 \\
16 \\
17 \\
18 \\
19 \\
20 \\
21 \\
22 \\
23 \\
24 \\
25 \\
26 \\
27 \\
28\end{array}$ & $\begin{array}{l}18 \cdot 0 \\
22 \cdot 5 \\
18.0 \\
40 \cdot 5 \\
72.0 \\
22 \cdot 5 \\
31.5 \\
31.5 \\
9 \cdot 0 \\
40.5 \\
22.5 \\
36.0 \\
13.5 \\
18.0 \\
13.5 \\
13.5 \\
22.5 \\
18.0 \\
18.0 \\
22.5 \\
9.0 \\
13.5\end{array}$ & \begin{tabular}{|r|}
21.3 \\
7.1 \\
0.0 \\
14.2 \\
21.3 \\
14.2 \\
7.1 \\
7.1 \\
0.0 \\
28.4 \\
0.0 \\
14.2 \\
0.0 \\
21.3 \\
7.1 \\
14.2 \\
7.1 \\
14.2 \\
28.4 \\
0.0 \\
0.0 \\
7.1
\end{tabular} & $\begin{array}{r}5 \cdot 8 \\
11 \cdot 6 \\
0 \cdot 0 \\
11.6 \\
0 \cdot 0 \\
5.8 \\
0 \cdot 0 \\
0 \cdot 0 \\
0 \cdot 0 \\
0 \cdot 0 \\
5 \cdot 8 \\
0 \cdot 0 \\
0 \cdot 0 \\
0 \cdot 0 \\
0 \cdot 0 \\
0 \cdot 0 \\
0 \cdot 0 \\
0 \cdot 0 \\
0 \cdot 0 \\
0 \cdot 0 \\
5.8 \\
0.0\end{array}$ & \begin{tabular}{|r|}
22.0 \\
13.5 \\
27.0 \\
22.5 \\
27.0 \\
4.5 \\
27.0 \\
$9 \cdot 0$ \\
$13 \cdot 5$ \\
$13 \cdot 5$ \\
$18 \cdot 0$ \\
22.5 \\
22.5 \\
$18 \cdot 0$ \\
9.0 \\
$18 \cdot 0$ \\
$13 \cdot 0$ \\
27.5 \\
22.5 \\
27.0
\end{tabular} & \begin{tabular}{r|}
35.5 \\
14.2 \\
74.1 \\
14.2 \\
14.2 \\
00.0 \\
0.0 \\
00.0 \\
0.0 \\
14.2 \\
21.3 \\
7.1 \\
0.0 \\
7.1 \\
0.0 \\
7.1 \\
7.1 \\
0.0 \\
14.2 \\
7.1 \\
14.2 \\
7.1
\end{tabular} & \begin{tabular}{r|}
0.0 \\
0.0 \\
0.0 \\
0.0 \\
0.0 \\
0.0 \\
5.8 \\
5.8 \\
5.8 \\
0.0 \\
5.8 \\
0.0 \\
5.8 \\
0.0 \\
0.0 \\
0.0 \\
0.0 \\
0.0 \\
0.0 \\
0.0 \\
0.0 \\
11.6
\end{tabular} & \begin{tabular}{|r|}
22.5 \\
22.5 \\
18.0 \\
27.0 \\
18.0 \\
18.0 \\
9.0 \\
22.5 \\
4.5 \\
9.0 \\
9.0 \\
22.5 \\
13.5 \\
22.5 \\
9.0 \\
0.0 \\
22.5 \\
18.0 \\
18.0 \\
22.5 \\
22.5 \\
13.5
\end{tabular} & \begin{tabular}{|r}
14.2 \\
7.1 \\
7.1 \\
14.2 \\
0.0 \\
0.0 \\
0.0 \\
28.4 \\
0.0 \\
7.1 \\
0.0 \\
7.1 \\
0.0 \\
14.2 \\
0.0 \\
21.3 \\
7.1 \\
0.0 \\
14.2 \\
7.1 \\
0.0 \\
0.0
\end{tabular} & \begin{tabular}{|r|}
0.0 \\
5.8 \\
0.0 \\
5.8 \\
0.0 \\
11.6 \\
0.0 \\
0.0 \\
0.0 \\
0.0 \\
0.0 \\
0.0 \\
0.0 \\
5.8 \\
0.0 \\
0.0 \\
0.0 \\
0.0 \\
0.0 \\
0.0 \\
5.8 \\
0.0
\end{tabular} & \begin{tabular}{|r|}
9.0 \\
13.0 \\
13.5 \\
13.5 \\
9.0 \\
4.5 \\
13.5 \\
0.0 \\
13.5 \\
13.5 \\
9.0 \\
13.5 \\
22.5 \\
0.0 \\
0.0 \\
4.5 \\
9.0 \\
22.5 \\
22.5 \\
22.5 \\
13.5
\end{tabular} & 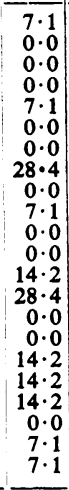 & \begin{tabular}{|r|}
0.0 \\
0.0 \\
5.8 \\
0.0 \\
5.8 \\
0.0 \\
5.8 \\
0.0 \\
11.6 \\
0.0 \\
0.0 \\
0.0 \\
11.6 \\
0.0 \\
0.0 \\
0.0 \\
0.0 \\
0.0 \\
0.0 \\
0.0 \\
11.6 \\
0.0
\end{tabular} & \begin{tabular}{|r|}
$9 \cdot 0$ \\
9.0 \\
4.5 \\
$27 \cdot 0$ \\
40.5 \\
$18 \cdot 0$ \\
$18 \cdot 0$ \\
$9 \cdot 0$ \\
4.5 \\
4.5 \\
$13 \cdot 5$ \\
4.5 \\
4.5 \\
0.0 \\
4.5 \\
0.0 \\
0.0 \\
9.0 \\
9.0 \\
4.5 \\
4.5 \\
0.0
\end{tabular} & 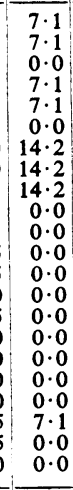 & \begin{tabular}{|l|}
$5 \cdot 8$ \\
$0 \cdot 0$ \\
$0 \cdot 0$ \\
$0 \cdot 0$ \\
$0 \cdot 0$ \\
$0 \cdot 0$ \\
$0 \cdot 0$ \\
$0 \cdot 0$ \\
$0 \cdot 0$ \\
$0 \cdot 0$ \\
$5 \cdot 8$ \\
$0 \cdot 0$ \\
$0 \cdot 0$ \\
$0 \cdot 0$ \\
$0 \cdot 0$ \\
$0 \cdot 0$ \\
$0 \cdot 0$ \\
$0 \cdot 0$ \\
$0 \cdot 0$ \\
$0 \cdot 0$ \\
$0 \cdot 0$ \\
0.0
\end{tabular} & \begin{tabular}{|r|}
4.5 \\
4.5 \\
9.0 \\
4.5 \\
4.5 \\
4.5 \\
9.0 \\
27.0 \\
9.0 \\
13.5 \\
0.0 \\
13.5 \\
0.0 \\
9.0 \\
4.5 \\
18.0 \\
13.5 \\
4.5 \\
13.5 \\
4.5 \\
0.0 \\
4.5
\end{tabular} & $\begin{array}{r}21.3 \\
0.0 \\
0.0 \\
0.0 \\
7.1 \\
14.2 \\
14.2 \\
0.0 \\
0.0 \\
0.0 \\
7.1 \\
7.1 \\
14.2 \\
7.1 \\
0.0 \\
7.1 \\
0.0 \\
7.1 \\
0.0 \\
7.1 \\
0.0 \\
0.0\end{array}$ & $\begin{array}{l}0 \\
0 \\
0 \\
0 \\
0 \\
0 \\
0 \\
5 \\
0 \\
0 \\
5\end{array}$ & $\begin{array}{r}13 \cdot 5 \\
0 \cdot 0 \\
0 \cdot 0 \\
18 \cdot 0 \\
9 \cdot 0 \\
0 \cdot 0 \\
4 \cdot 5 \\
9 \cdot 0 \\
13 \cdot 5 \\
0 \cdot 0 \\
0 \cdot 0 \\
4 \cdot 5 \\
4 \cdot 5 \\
9 \cdot 0 \\
9 \cdot 0 \\
9 \cdot 0 \\
4 \cdot 5 \\
0 \cdot 0 \\
4 \cdot 5 \\
9 \cdot 0 \\
0 \cdot 0 \\
0 \cdot 0\end{array}$ & $\begin{array}{r}0.0 \\
7.1 \\
0.0 \\
7.1 \\
0.0 \\
0.0 \\
00.0 \\
0.0 \\
0.0 \\
0.0 \\
0.0 \\
7.1 \\
7.1 \\
7.1 \\
0.0 \\
0.0 \\
7.1 \\
0.0 \\
0.0 \\
14.2 \\
0.0 \\
0.0\end{array}$ & 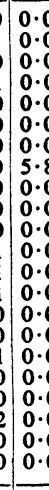 & \\
\hline ransit & $\begin{array}{l}29 \\
30 \\
31 \\
32 \\
33\end{array}$ & $\begin{array}{l}0 \cdot 0 \\
4 \cdot 5 \\
9 \cdot 0 \\
0 \cdot 0 \\
0 \cdot 0\end{array}$ & \begin{tabular}{|l|}
0.0 \\
7.1 \\
0.0 \\
0.0 \\
0.0
\end{tabular} & $\begin{array}{l}0 \cdot 0 \\
0 \cdot 0 \\
0 \cdot 0 \\
0 \cdot 0 \\
0 \cdot 0\end{array}$ & $\begin{array}{r}22.5 \\
0.0 \\
13.5 \\
0.0 \\
0.0\end{array}$ & \begin{tabular}{|r|}
$14 \cdot 2$ \\
$0 \cdot 0$ \\
$14 \cdot 2$ \\
$7 \cdot 1$ \\
$0 \cdot 0$
\end{tabular} & $\begin{array}{c}11.6 \\
0.0 \\
0.0 \\
0.0 \\
0.0\end{array}$ & $\begin{array}{r}13 \cdot 5 \\
0 \cdot 0 \\
36 \cdot 0 \\
4 \cdot 5 \\
0 \cdot 0\end{array}$ & $\begin{array}{r}0 \cdot 0 \\
7 \cdot 1 \\
0 \cdot 0 \\
14 \cdot 2 \\
0.0\end{array}$ & $\begin{array}{l}0 \cdot 0 \\
0 \cdot 0 \\
5 \cdot 8 \\
0 \cdot 0 \\
0 \cdot 0\end{array}$ & $\begin{array}{r}13 \cdot 5 \\
0.0 \\
9 \cdot 0 \\
0.0 \\
0.0\end{array}$ & $\begin{array}{l}7.1 \\
0.0 \\
0.0 \\
7.1 \\
0.0\end{array}$ & $\begin{array}{l}5 \cdot 8 \\
0.0 \\
0.0 \\
0.0 \\
0.0\end{array}$ & $\begin{array}{r}13 \cdot 5 \\
4 \cdot 5 \\
13 \cdot 5 \\
0 \cdot 0 \\
0 \cdot 0\end{array}$ & 占 & & $\begin{array}{l}4.5 \\
4.5 \\
4.5 \\
0.0\end{array}$ & $\begin{array}{c}0 \cdot 0 \\
0.0 \\
14 \cdot 2 \\
0.0\end{array}$ & & $\begin{array}{r}27.0 \\
0.0 \\
0.0 \\
0.0 \\
0.0\end{array}$ & $\begin{array}{r}14 \cdot 2 \\
7.1 \\
0.0 \\
0.0 \\
0.0\end{array}$ & $\begin{array}{l}2 \\
1 \\
0 \\
0 \\
0\end{array}$ & \\
\hline
\end{tabular}

TABLE

THE INFLUENCE OF

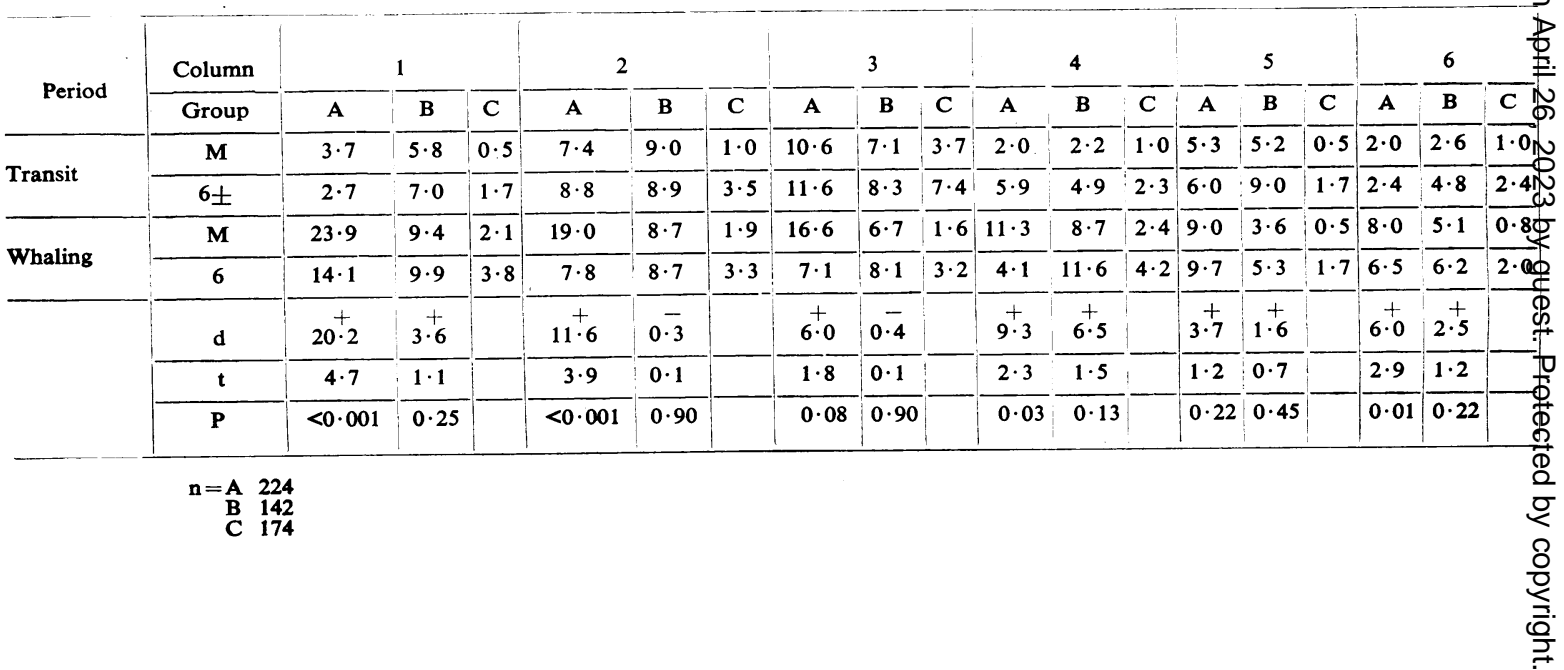


1,000 for Classified Diseases

\begin{tabular}{|c|c|c|c|c|c|c|c|c|c|c|c|c|c|c|c|c|c|c|c|c|c|c|c|c|}
\hline \multicolumn{3}{|c|}{$\begin{array}{c}\text { Venereal } \\
\text { disease } \\
8\end{array}$} & \multicolumn{3}{|c|}{$\begin{array}{c}\text { Diseases of } \\
\text { upper } \\
\text { respiratory } \\
\text { tract } \\
9\end{array}$} & \multicolumn{3}{|c|}{$\begin{array}{c}\text { Burns and } \\
\text { scalds } \\
10\end{array}$} & \multicolumn{3}{|c|}{$\begin{array}{c}\begin{array}{c}\text { Dental } \\
\text { conditions }\end{array} \\
11\end{array}$} & \multicolumn{3}{|c|}{$\begin{array}{c}\text { Disorders } \\
\text { of C.N.S. } \\
12\end{array}$} & \multicolumn{3}{|c|}{$\begin{array}{c}\text { Diarrhoea } \\
13\end{array}$} & \multicolumn{3}{|c|}{$\begin{array}{c}\text { Miscellaneous } \\
\text { conditions } \\
14\end{array}$} & \multicolumn{3}{|c|}{$\begin{array}{c}\text { Total classified } \\
\text { weekly morbidity } \\
\text { rate }\end{array}$} & \multirow[t]{2}{*}{$\begin{array}{c}\text { Total } \\
\text { weekly } \\
\text { morbidi- } \\
\text { ity rate }\end{array}$} \\
\hline $\mathbf{A}$ & B & C & $\mathbf{A}$ & B & C & $\mathbf{A}$ & B & C & $\mathbf{A}$ & B & C & $\mathbf{A}$ & B & C & A & B & $\mathrm{C}$ & $\mathbf{A}$ & B & $C$ & $\mathbf{A}$ & B & C & \\
\hline $\begin{array}{r}0.0 \\
0.0 \\
18 \cdot 0 \\
22.5 \\
9.0 \\
0.0\end{array}$ & $\begin{array}{l}0.0 \\
0.0 \\
0.0 \\
7.1 \\
7.1 \\
0.0\end{array}$ & $\begin{array}{l}5.8 \\
0.0 \\
5.8 \\
5.8 \\
0.0 \\
0.0\end{array}$ & $\begin{array}{l}0.0 \\
4.5 \\
4.5 \\
9 \cdot 0 \\
4.5 \\
0.0\end{array}$ & $\begin{array}{l}0.0 \\
0.0 \\
7 \cdot 1 \\
7.1 \\
0.0 \\
0.0\end{array}$ & \begin{tabular}{|l|}
0.0 \\
0.0 \\
0.0 \\
5.8 \\
0.0 \\
5.8
\end{tabular} & $\begin{array}{l}0 \cdot 0 \\
4 \cdot 5 \\
0 \cdot 0 \\
0 \cdot 0 \\
4 \cdot 5 \\
4 \cdot 5\end{array}$ & $\begin{array}{l}0 \cdot 0 \\
0 \cdot 0 \\
7 \cdot 1 \\
0 \cdot 0 \\
7 \cdot 1 \\
0 \cdot 0\end{array}$ & \begin{tabular}{l|l}
$0 \cdot 0$ \\
$0 \cdot 0$ \\
$0 \cdot 0$ \\
$0 \cdot 0$ \\
$0 \cdot 0$ \\
$0 \cdot 0$
\end{tabular} & $\begin{array}{l}0.0 \\
4.5 \\
0.0 \\
0 \cdot 0 \\
0.0 \\
4.5\end{array}$ & $\begin{array}{l}0.0 \\
0.0 \\
0.0 \\
0.0 \\
0.0 \\
0.0\end{array}$ & & $\begin{array}{l}0 \cdot 0 \\
0 \cdot 0 \\
0 \cdot 0 \\
0 \cdot 0 \\
0 \cdot 0 \\
0 \cdot 0\end{array}$ & \begin{tabular}{l|l}
$0 \cdot 0$ \\
0.0 \\
0.0 \\
0.0 \\
0.0 \\
0.0
\end{tabular} & \begin{tabular}{|l|}
0.0 \\
0.0 \\
5.8 \\
0.0 \\
0.0 \\
0.0
\end{tabular} & $\begin{array}{l}0.0 \\
0 \cdot 0 \\
4.5 \\
4.5 \\
0.0 \\
0.0\end{array}$ & $\mid \begin{array}{l}0 \cdot 0 \\
0 \cdot 0 \\
0 \cdot 0 \\
0 \cdot 0 \\
0 \cdot 0 \\
0 \cdot 0\end{array}$ & $\begin{array}{l}0.0 \\
0.0 \\
0 \cdot 0 \\
5.8 \\
0 \cdot 0 \\
5.8\end{array}$ & \begin{tabular}{l|}
0.0 \\
0.0 \\
0.0 \\
0.0 \\
0.0 \\
4.5
\end{tabular} & \begin{tabular}{|r|}
7.1 \\
0.0 \\
0.0 \\
21.3 \\
0.0 \\
0.0
\end{tabular} & \begin{tabular}{|l|}
$5 \cdot 8$ \\
0.0 \\
0.0 \\
0.0 \\
0.0 \\
0.0
\end{tabular} & \begin{tabular}{|r|}
4.5 \\
31.5 \\
45.0 \\
94.5 \\
85.5 \\
58.5
\end{tabular} & $\begin{array}{l}21 \cdot 3 \\
7 \cdot 1 \\
85 \cdot 2 \\
92 \cdot 3 \\
99 \cdot 4 \\
56 \cdot 8\end{array}$ & $\begin{array}{l}11 \cdot 6 \\
5.8 \\
17.4 \\
40.6 \\
29.0 \\
17.4\end{array}$ & $\begin{array}{l}11 \cdot 2 \\
16 \cdot 7 \\
46 \cdot 5 \\
76 \cdot 3 \\
70 \cdot 7 \\
44.6\end{array}$ \\
\hline $\begin{array}{r}13.5 \\
0.0 \\
0.0 \\
0.0 \\
9.0 \\
0.0 \\
4.5 \\
4.5 \\
4.5 \\
9.0 \\
0.0 \\
4.5 \\
0.0 \\
4.5 \\
4.5 \\
0.0 \\
0.0 \\
0.0 \\
0.0 \\
4.5 \\
0.0 \\
0.0\end{array}$ & $\begin{array}{c}14.2 \\
0.0 \\
7.1 \\
0.0 \\
0.0 \\
0.0 \\
7.1 \\
7.1 \\
0.0 \\
0.0 \\
0.0 \\
0.0 \\
0.0 \\
0.0 \\
0.0 \\
0.0 \\
0.0 \\
0.0 \\
0.0 \\
0.0 \\
0.0 \\
0.0\end{array}$ & \begin{tabular}{|c|}
11.6 \\
5.8 \\
0.0 \\
0.0 \\
0.0 \\
5.8 \\
0.0 \\
5.8 \\
0.0 \\
0.0 \\
0.0 \\
0.0 \\
0.0 \\
0.0 \\
0.0 \\
0.0 \\
0.0 \\
0.0 \\
0.0 \\
0.0 \\
0.0 \\
0.0
\end{tabular} & $\begin{array}{r}4 \cdot 5 \\
9 \cdot 0 \\
4 \cdot 5 \\
4 \cdot 5 \\
0 \cdot 0 \\
0 \cdot 0 \\
0 \cdot 0 \\
0 \cdot 0 \\
4 \cdot 5 \\
0 \cdot 0 \\
0 \cdot 0 \\
4 \cdot 5 \\
0 \cdot 0 \\
13 \cdot 5 \\
0 \cdot 0 \\
4 \cdot 5 \\
4 \cdot 5 \\
13 \cdot 5 \\
0 \cdot 0 \\
0 \cdot 0 \\
0 \cdot 0 \\
0 \cdot 0\end{array}$ & $\begin{array}{r}14.2 \\
0.0 \\
7.1 \\
7.1 \\
14.2 \\
0.0 \\
0.0 \\
7.1 \\
0.0 \\
0.0 \\
0.0 \\
0.0 \\
7.1 \\
0.0 \\
7.1 \\
0.0 \\
0.0 \\
7.1 \\
0.0 \\
0.0 \\
0.0 \\
0.0\end{array}$ & $\mid \begin{array}{l}0 \cdot 0 \\
0 \cdot 0 \\
0.0 \\
0 \cdot 0 \\
0 \cdot 0 \\
0 \cdot 0 \\
0 \cdot 0 \\
0 \cdot 0 \\
0 \cdot 0 \\
0 \cdot 0 \\
0 \cdot 0 \\
0 \cdot 0 \\
0 \cdot 0 \\
0 \cdot 0 \\
0 \cdot 0 \\
0 \cdot 0 \\
0 \cdot 0 \\
0 \cdot 0 \\
0 \cdot 0 \\
0 \cdot 0 \\
0 \cdot 0 \\
0.0\end{array}$ & $\begin{array}{l}4 \cdot 5 \\
0 \cdot 0 \\
0 \cdot 0 \\
4 \cdot 5 \\
4 \cdot 5 \\
0 \cdot 0 \\
0 \cdot 0 \\
9 \cdot 0 \\
0 \cdot 0 \\
4 \cdot 5 \\
0 \cdot 0 \\
0.0 \\
0 \cdot 0 \\
0 \cdot 0 \\
4 \cdot 5 \\
4.5 \\
0 \cdot 0 \\
4 \cdot 5 \\
0 \cdot 0 \\
4 \cdot 5 \\
0 \cdot 0 \\
0 \cdot 0\end{array}$ & $\begin{array}{r}14.2 \\
7.1 \\
0.0 \\
0.0 \\
7.1 \\
0.0 \\
0.0 \\
7.1 \\
14.2 \\
0.0 \\
0.0 \\
0.0 \\
0.0 \\
7.1 \\
0.0 \\
0.0 \\
0.0 \\
0.0 \\
7.1 \\
0.0 \\
0.0 \\
7.1\end{array}$ & $\begin{array}{l}0 \cdot 0 \\
0 \cdot 0 \\
0 \cdot 0 \\
0 \cdot 0 \\
0 \cdot 0 \\
0 \cdot 0 \\
5 \cdot 0 \\
0 \cdot 0 \\
0 \cdot 0 \\
0 \cdot 0 \\
0 \cdot 0 \\
0 \cdot 0 \\
0 \cdot 0 \\
0 \cdot 0 \\
5 \cdot 0 \\
0 \cdot 0 \\
0 \cdot 0 \\
0 \cdot 0 \\
0 \cdot 0 \\
0 \cdot 0 \\
0 \cdot 0 \\
0 \cdot 0\end{array}$ & $\begin{array}{r}0 \cdot 0 \\
4.5 \\
0 \cdot 0 \\
0 \cdot 0 \\
13.5 \\
0 \cdot 0 \\
0 \cdot 0 \\
9 \cdot 0 \\
4.5 \\
9 \cdot 0 \\
9 \cdot 0 \\
4.5 \\
4.5 \\
9 \cdot 0 \\
0 \cdot 0 \\
4.5 \\
0 \cdot 0 \\
0 \cdot 0 \\
4 \cdot 5 \\
4 \cdot 5 \\
9 \cdot 0 \\
0 \cdot 0\end{array}$ & $\begin{array}{c}7.1 \\
0.0 \\
0.0 \\
0.0 \\
0.0 \\
0.0 \\
0.0 \\
0.0 \\
7.0 \\
7.1 \\
0.0 \\
0.0 \\
0.0 \\
0.0 \\
0.0 \\
0.0 \\
0.0 \\
0.0 \\
0.0 \\
0.0 \\
7.1 \\
0.0 \\
14.2\end{array}$ & $\begin{array}{l}0 \cdot 0 \\
0 \cdot 0 \\
0 \cdot 0 \\
0 \cdot 0 \\
0 \cdot 0 \\
0 \cdot 0 \\
0 \cdot 0 \\
0 \cdot 0 \\
0 \cdot 0 \\
0 \cdot 0 \\
0 \cdot 0 \\
5 \cdot 0 \\
0 \cdot 0 \\
0 \cdot 0 \\
0 \cdot 0 \\
0 \cdot 0 \\
0 \cdot 0 \\
0 \cdot 0 \\
0 \cdot 0 \\
0 \cdot 0 \\
0 \cdot 0 \\
0.0\end{array}$ & $\begin{array}{l}0 \cdot 0 \\
0 \cdot 0 \\
0 \cdot 0 \\
9 \cdot 0 \\
0 \cdot 0 \\
0 \cdot 0 \\
0 \cdot 0 \\
9 \cdot 0 \\
0 \cdot 0 \\
0 \cdot 0 \\
0 \cdot 0 \\
0 \cdot 0 \\
0 \cdot 0 \\
0 \cdot 0 \\
4.5 \\
0 \cdot 0 \\
0 \cdot 0 \\
0 \cdot 0 \\
9 \cdot 0 \\
9 \cdot 0 \\
0 \cdot 0 \\
4 \cdot 5\end{array}$ & $\mid \begin{array}{l}0 \cdot 0 \\
0 \cdot 0 \\
0 \cdot 0 \\
0.0 \\
0 \cdot 0 \\
0 \cdot 0 \\
0.0 \\
7 \cdot 1 \\
7 \cdot 1 \\
0 \cdot 0 \\
7 \cdot 1 \\
0 \cdot 0 \\
0 \cdot 0 \\
0 \cdot 0 \\
0 \cdot 0 \\
7 \cdot 1 \\
0 \cdot 0 \\
0 \cdot 0 \\
0.0 \\
7 \cdot 1 \\
7 \cdot 1 \\
0 \cdot 0\end{array}$ & $\begin{array}{l}0 \cdot 0 \\
0 \cdot 0 \\
0 \cdot 0 \\
0 \cdot 0 \\
0 \cdot 0 \\
0 \cdot 0 \\
0 \cdot 0 \\
0 \cdot 0 \\
0 \cdot 0 \\
0 \cdot 0 \\
0 \cdot 0 \\
0 \cdot 0 \\
5 \cdot 8 \\
0 \cdot 0 \\
5 \cdot 8 \\
0 \cdot 0 \\
5 \cdot 0 \\
0 \cdot 0 \\
0 \cdot 0 \\
0 \cdot 0 \\
0 \cdot 0 \\
0 \cdot 0\end{array}$ & $\begin{array}{l}4 \cdot 5 \\
0 \cdot 0 \\
0 \cdot 0 \\
0 \cdot 0 \\
0 \cdot 0 \\
0 \cdot 0 \\
0 \cdot 0 \\
4 \cdot 5 \\
0 \cdot 0 \\
0 \cdot 0 \\
4.5 \\
0 \cdot 0 \\
0 \cdot 0 \\
0 \cdot 0 \\
0 \cdot 0 \\
0 \cdot 0 \\
0 \cdot 0 \\
0 \cdot 0 \\
9 \cdot 0 \\
0 \cdot 0 \\
0.0 \\
0 \cdot 0\end{array}$ & $\begin{array}{l}7 \cdot 1 \\
0 \cdot 0 \\
0 \cdot 0 \\
0 \cdot 0 \\
0 \cdot 0 \\
0 \cdot 0 \\
7 \cdot 0 \\
0 \cdot 0 \\
0 \cdot 0 \\
0 \cdot 0 \\
0 \cdot 0 \\
0 \cdot 0 \\
0 \cdot 0 \\
0 \cdot 0 \\
0 \cdot 0 \\
0 \cdot 0 \\
0 \cdot 0 \\
0 \cdot 0 \\
0 \cdot 0 \\
0 \cdot 0 \\
0 \cdot 0 \\
0 \cdot 0\end{array}$ & $\begin{array}{l}0 \cdot 0 \\
0 \cdot 0 \\
0 \cdot 0 \\
0 \cdot 0 \\
0 \cdot 0 \\
0 \cdot 0 \\
0 \cdot 0 \\
0 \cdot 0 \\
0 \cdot 0 \\
0 \cdot 0 \\
0 \cdot 0 \\
0 \cdot 0 \\
0 \cdot 0 \\
0 \cdot 0 \\
0 \cdot 0 \\
0 \cdot 0 \\
0 \cdot 0 \\
0 \cdot 0 \\
0 \cdot 0 \\
0 \cdot 0 \\
0 \cdot 0 \\
0 \cdot 0\end{array}$ & \begin{tabular}{|c|}
9.0 \\
0.0 \\
4.5 \\
4.5 \\
13.5 \\
9.0 \\
9.0 \\
0.0 \\
4.5 \\
13.5 \\
0.0 \\
4.5 \\
0.0 \\
9.0 \\
0.0 \\
4.5 \\
0.0 \\
9.0 \\
9.0 \\
4.5 \\
0.0 \\
0.0 \\
\end{tabular} & \begin{tabular}{|l|}
$7 \cdot 1$ \\
$7 \cdot 1$ \\
$0 \cdot 0$ \\
0.0 \\
7.1 \\
$0 \cdot 0$ \\
$0 \cdot 0$ \\
$0 \cdot 0$ \\
$0 \cdot 0$ \\
$0 \cdot 0$ \\
$0 \cdot 0$ \\
$0 \cdot 0$ \\
$7 \cdot 1$ \\
7.1 \\
$0 \cdot 0$ \\
$7 \cdot 1$ \\
7.1 \\
$0 \cdot 0$ \\
7.1 \\
0.0 \\
$0 \cdot 0$ \\
$7 \cdot 1$
\end{tabular} & $\begin{array}{l}0 \cdot 0 \\
5 \cdot 8 \\
0.0 \\
5.8 \\
0 \cdot 0 \\
0 \cdot 0 \\
0 \cdot 0 \\
0 \cdot 0 \\
0 \cdot 0 \\
0 \cdot 0 \\
0 \cdot 0 \\
0 \cdot 0 \\
0 \cdot 0 \\
0 \cdot 0 \\
0 \cdot 0 \\
5.8 \\
0 \cdot 0 \\
0 \cdot 0 \\
0 \cdot 0 \\
0 \cdot 0 \\
0 \cdot 0 \\
0.0\end{array}$ & \begin{tabular}{|c|}
121.5 \\
103.5 \\
85.5 \\
$180 \cdot 0$ \\
216.0 \\
$108 \cdot 0$ \\
94.5 \\
175.5 \\
67.5 \\
130.5 \\
85.5 \\
126.0 \\
76.5 \\
135.0 \\
72.0 \\
67.5 \\
$90 \cdot 0$ \\
$121 \cdot 5$ \\
130.5 \\
139.5 \\
$90 \cdot 0$ \\
76.5
\end{tabular} & \begin{tabular}{|c|}
$170 \cdot 4$ \\
$56 \cdot 8$ \\
$28 \cdot 4$ \\
63.9 \\
$85 \cdot 2$ \\
$28 \cdot 4$ \\
$49 \cdot 7$ \\
$106 \cdot 5$ \\
$42 \cdot 6$ \\
$63 \cdot 9$ \\
$35 \cdot 5$ \\
$42 \cdot 6$ \\
$49 \cdot 7$ \\
$99 \cdot 4$ \\
$14 \cdot 2$ \\
$63 \cdot 9$ \\
$49 \cdot 7$ \\
$42 \cdot 6$ \\
$92 \cdot 3$ \\
$56 \cdot 8$ \\
$28 \cdot 4$ \\
$49 \cdot 7$
\end{tabular} & \begin{tabular}{|c|}
23.2 \\
29.0 \\
5.8 \\
23.2 \\
5.8 \\
23.2 \\
17.4 \\
23.2 \\
17.4 \\
0.0 \\
23.2 \\
11.6 \\
23.2 \\
5.8 \\
11.6 \\
5.8 \\
5.8 \\
0.0 \\
0.0 \\
0.0 \\
23.2 \\
11.6
\end{tabular} & $\begin{array}{r}102.3 \\
67.0 \\
44.6 \\
98.6 \\
113.5 \\
59.5 \\
57.7 \\
107.9 \\
44.6 \\
70.7 \\
52.1 \\
67.0 \\
52.1 \\
83.7 \\
37.2 \\
46.5 \\
52.1 \\
61.4 \\
78.4 \\
72.5 \\
52.1 \\
48.4\end{array}$ \\
\hline $\begin{array}{r}0.0 \\
13.5 \\
4.5 \\
0 \cdot 0 \\
0.0\end{array}$ & $\begin{array}{l}0.0 \\
7.1 \\
7.1 \\
0.0 \\
0.0\end{array}$ & $\begin{array}{l}0 \cdot 0 \\
0 \cdot 0 \\
0 \cdot 0 \\
0 \cdot 0 \\
0 \cdot 0\end{array}$ & $\begin{array}{l}4.5 \\
0.0 \\
4.5 \\
0.0 \\
0.0\end{array}$ & $\begin{array}{l}0 \cdot 0 \\
7 \cdot 1 \\
0 \cdot 0 \\
0 \cdot 0 \\
0 \cdot 0\end{array}$ & $\left|\begin{array}{l}0 \cdot 0 \\
0 \cdot 0 \\
0 \cdot 0 \\
0 \cdot 0 \\
0 \cdot 0\end{array}\right|$ & $\begin{array}{r}18 \cdot 0 \\
0 \cdot 0 \\
0 \cdot 0 \\
0 \cdot 0 \\
0 \cdot 0\end{array}$ & $\begin{array}{l}7 \cdot 1 \\
0 \cdot 0 \\
7 \cdot 1 \\
0 \cdot 0 \\
0 \cdot 0\end{array}$ & $\begin{array}{l}0 \cdot 0 \\
0 \cdot 0 \\
0 \cdot 0 \\
0 \cdot 0 \\
0 \cdot 0\end{array}$ & $\begin{array}{l}4.5 \\
0 \cdot 0 \\
0 \cdot 0 \\
0 \cdot 0 \\
0.0\end{array}$ & $\begin{array}{l}0.0 \\
0.0 \\
0.0 \\
0.0 \\
0.0\end{array}$ & $\begin{array}{l}0 \cdot 0 \\
0 \cdot 0 \\
0 \cdot 0 \\
0 \cdot 0 \\
0.0\end{array}$ & $\mid \begin{array}{l}0.0 \\
0.0 \\
4.5 \\
4.5 \\
0.0\end{array}$ & $\mid$\begin{tabular}{l|}
$0 \cdot 0$ \\
$0 \cdot 0$ \\
$7 \cdot 1$ \\
$0 \cdot 0$ \\
$0 \cdot 0$
\end{tabular} & $\begin{array}{l}0 \cdot 0 \\
0 \cdot 0 \\
0 \cdot 0 \\
0 \cdot 0 \\
0 \cdot 0\end{array}$ & $\begin{array}{l}0 \cdot 0 \\
4 \cdot 5 \\
0 \cdot 0 \\
4 \cdot 5 \\
0.0\end{array}$ & $\begin{array}{l}0.0 \\
0.0 \\
0.0 \\
0.0 \\
0.0\end{array}$ & $\begin{array}{l}0.0 \\
5.8 \\
0.0 \\
0.0 \\
0.0\end{array}$ & $\begin{array}{l}0 \cdot 0 \\
0 \cdot 0 \\
9 \cdot 0 \\
4 \cdot 5 \\
0 \cdot 0\end{array}$ & $\begin{array}{r}14.2 \\
7.1 \\
7.1 \\
0.0 \\
0.0\end{array}$ & $\mid$\begin{tabular}{l|l}
$0 \cdot 0$ \\
0.0 \\
$0 \cdot 0$ \\
$0 \cdot 0$ \\
$0 \cdot 0$
\end{tabular} & \begin{tabular}{|r|}
117.0 \\
31.5 \\
108.0 \\
$18 \cdot 0$ \\
0.0
\end{tabular} & \begin{tabular}{|r|}
56.8 \\
42.6 \\
63.9 \\
28.4 \\
0.0
\end{tabular} & \begin{tabular}{r|}
17.4 \\
5.8 \\
5.8 \\
0.0 \\
0.0
\end{tabular} & $\begin{array}{r}68 \cdot 8 \\
26 \cdot 0 \\
63 \cdot 2 \\
14 \cdot 9 \\
0 \cdot 0\end{array}$ \\
\hline
\end{tabular}

VI

Whaling on Disease

\begin{tabular}{|c|c|c|c|c|c|c|c|c|c|c|c|c|c|c|c|c|c|c|c|c|c|c|c|c|}
\hline \multicolumn{3}{|c|}{7} & \multicolumn{3}{|c|}{8} & \multicolumn{3}{|c|}{9} & \multicolumn{3}{|c|}{10} & \multicolumn{3}{|c|}{11} & \multicolumn{3}{|c|}{12} & \multicolumn{3}{|c|}{13} & \multicolumn{2}{|c|}{ Total } & \multirow[b]{2}{*}{ C } & \multirow{2}{*}{$\begin{array}{c}\text { Un- } \\
\text { classi- } \\
\text { fied } \\
\text { Total }\end{array}$} \\
\hline $\mathbf{A}$ & B & C & $\mathbf{A}$ & B & C & A & B & C & $\mathbf{A}$ & B & C & $\mathbf{A}$ & B & C & $\mathbf{A}$ & B & C & $\mathbf{A}$ & B & C & $\mathbf{A}$ & B & & \\
\hline $5 \cdot 7$ & 3.9 & 0.0 & $5 \cdot 7$ & $2 \cdot 6$ & 1.6 & $2 \cdot 9$ & 1.9 & $1 \cdot 0$ & $2 \cdot 9$ & $2 \cdot 6$ & $1 \cdot 0$ & $1 \cdot 2$ & 0.0 & 0.5 & 0.8 & 0.6 & 0.5 & $1 \cdot 6$ & 0.0 & $1 \cdot 6$ & $54 \cdot 0$ & $50 \cdot 3$ & $13 \cdot 7$ & $37 \cdot 9$ \\
\hline $9 \cdot 0$ & 4.9 & 0.0 & $8 \cdot 6$ & $3 \cdot 6$ & $2 \cdot 7$ & $3 \cdot 0$ & $3 \cdot 3$ & 0.2 & $5 \cdot 4$ & $3 \cdot 6$ & 0.2 & $2 \cdot 1$ & 0.0 & $1 \cdot 7$ & 1.8 & $2 \cdot 1$ & $1 \cdot 7$ & $2 \cdot 3$ & 0.0 & $\overline{2 \cdot 7}$ & $41 \cdot 5$ & $34 \cdot 1$ & $12 \cdot 5$ & $27 \cdot 3$ \\
\hline $5 \cdot 5$ & $2 \cdot 6$ & 0.3 & 2.9 & $1 \cdot 6$ & $1 \cdot 3$ & $3 \cdot 1$ & $3 \cdot 3$ & 0.0 & $2 \cdot 0$ & $3 \cdot 3$ & 0.0 & $4 \cdot 1$ & 1.9 & $0 \cdot 3$ & $2 \cdot 1$ & $1 \cdot 9$ & 0.8 & $1 \cdot 0$ & 0.6 & 0.0 & $111 \cdot 3$ & $60 \cdot 0$ & $13 \cdot 2$ & 66.4 \\
\hline $5 \cdot 4$ & $4 \cdot 1$ & $1 \cdot 3$ & $3 \cdot 8$ & $3 \cdot 8$ & $3 \cdot 1$ & $4 \cdot 3$ & $4 \cdot 8$ & 0.0 & $2 \cdot 7$ & $4 \cdot 8$ & 0.0 & $4 \cdot 1$ & $4 \cdot 2$ & $1 \cdot 3$ & 3.6 & $3 \cdot 3$ & $2 \cdot 0$ & $2 \cdot 6$ & $2 \cdot 1$ & $\overline{0.0}$ & $39 \cdot 5$ & $34 \cdot 1$ & $11 \cdot 2$ & $22 \cdot 0$ \\
\hline $0 . \overline{2}$ & $\overline{1 \cdot 3}$ & & $2 \cdot \overline{8}$ & $1 \cdot \overline{0}$ & & $\begin{array}{c}+ \\
1 \cdot 4\end{array}$ & $\begin{array}{c}+ \\
1 \cdot 4\end{array}$ & & $0 . \overline{9}$ & $0 \cdot+$ & & $2 \cdot+$ & $\begin{array}{l}+ \\
1.9\end{array}$ & & $1+3$ & $1+3$ & & $0 . \overline{6}$ & $\begin{array}{l}+ \\
0.6\end{array}$ & & ${ }_{63 \cdot 3}^{+}$ & $9 \cdot+$ & & $\stackrel{+}{+}$ \\
\hline 0.1 & 0.8 & & $1 \cdot 3$ & 0.7 & & $0 \cdot 1$ & 0.9 & & 0.6 & 0.4 & & $2 \cdot 1$ & $1 \cdot 5$ & & $1 \cdot 1$ & $1 \cdot 2$ & & 0.6 & $1 \cdot 0$ & & $4 \cdot 0$ & 0.8 & & $2 \cdot 9$ \\
\hline 0.90 & 0.40 & & 0.25 & 0.45 & & 0.90 & 0.34 & & 0.52 & 0.66 & & 0.04 & $0 \cdot 13$ & & $\overline{0.25}$ & $\overline{0.22}$ & & 0.52 & 0.29 & & $<0.001$ & 0.40 & & 0.005 \\
\hline
\end{tabular}




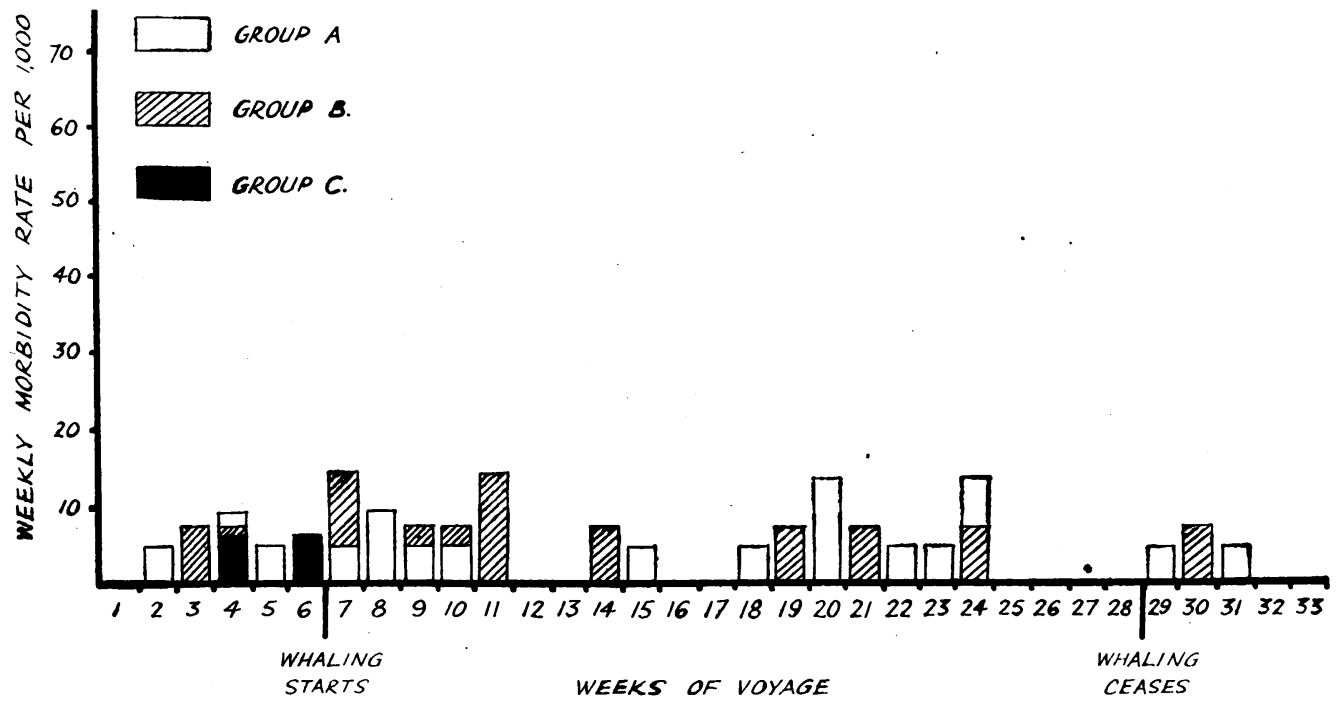

Fio. 9.-Weekly morbidity rate per 1,000 for each group for Column 9 (diseases of the upper respiratory tract).

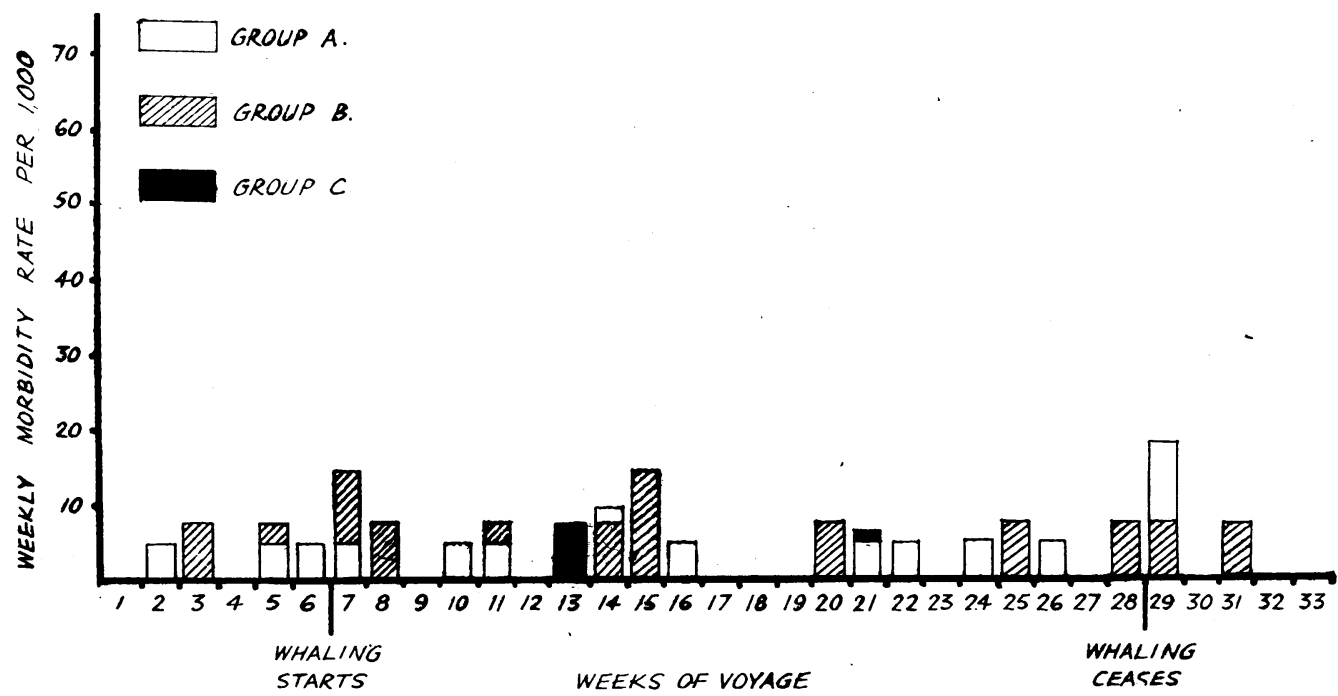

Fig. 10.-Weekly morbidity rate per 1,000 for each group for Column 10 (burns and scalds). 


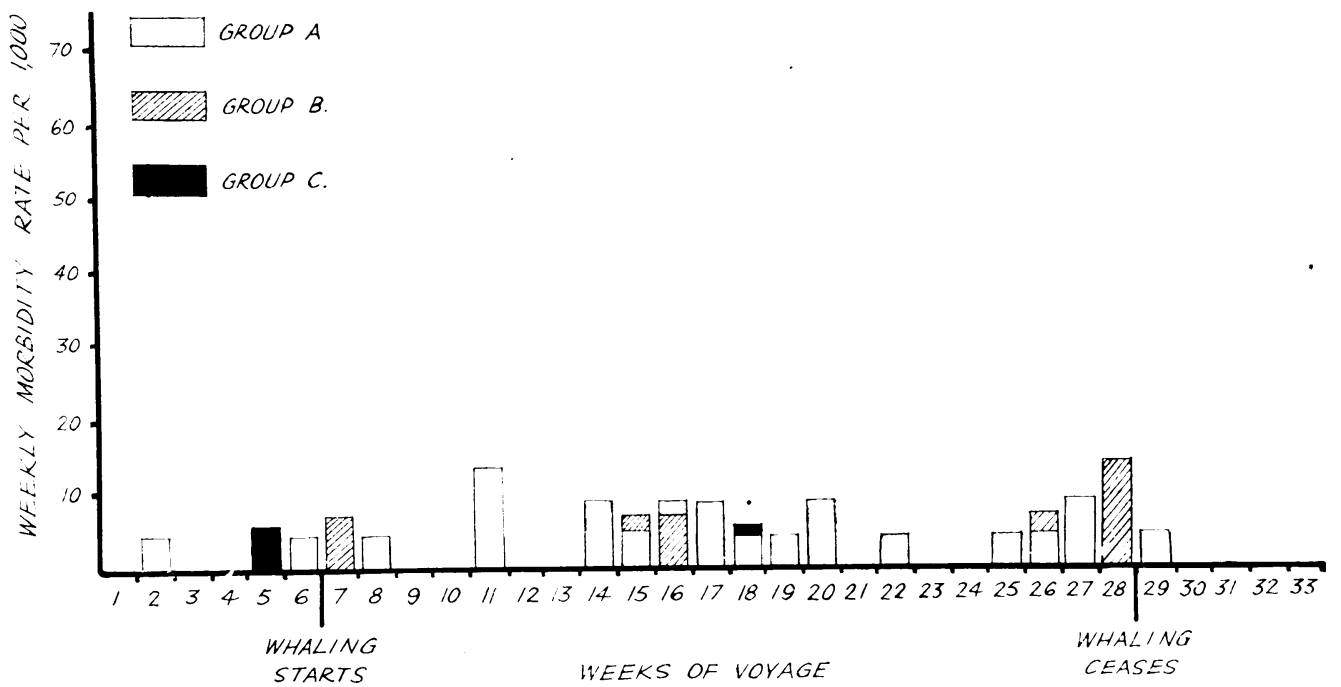

FIG. 11.-Weekly morbidity rate per 1,000 for each group for Column 11 (dental conditions).

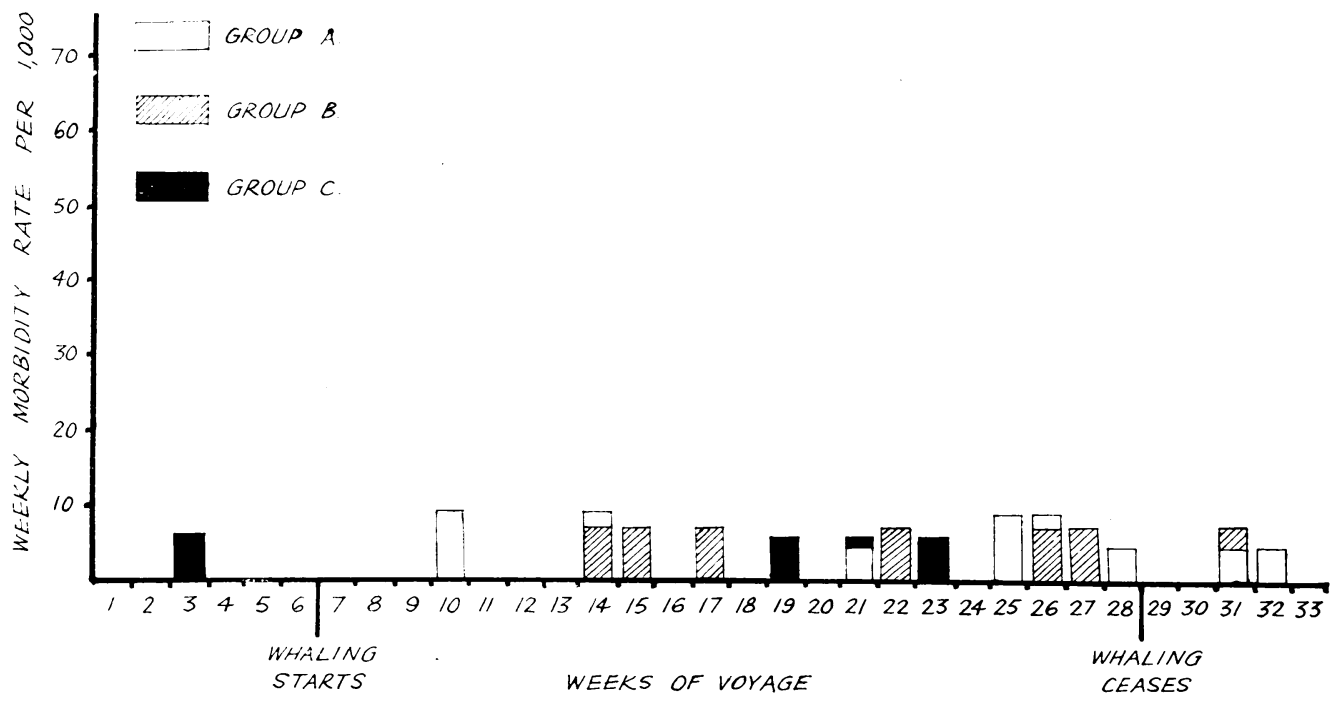

FIG. 12.-Weekly morbidity rate per 1,000 for each group for Column 12 (disorders of the central nervous system (organic and functional).) 


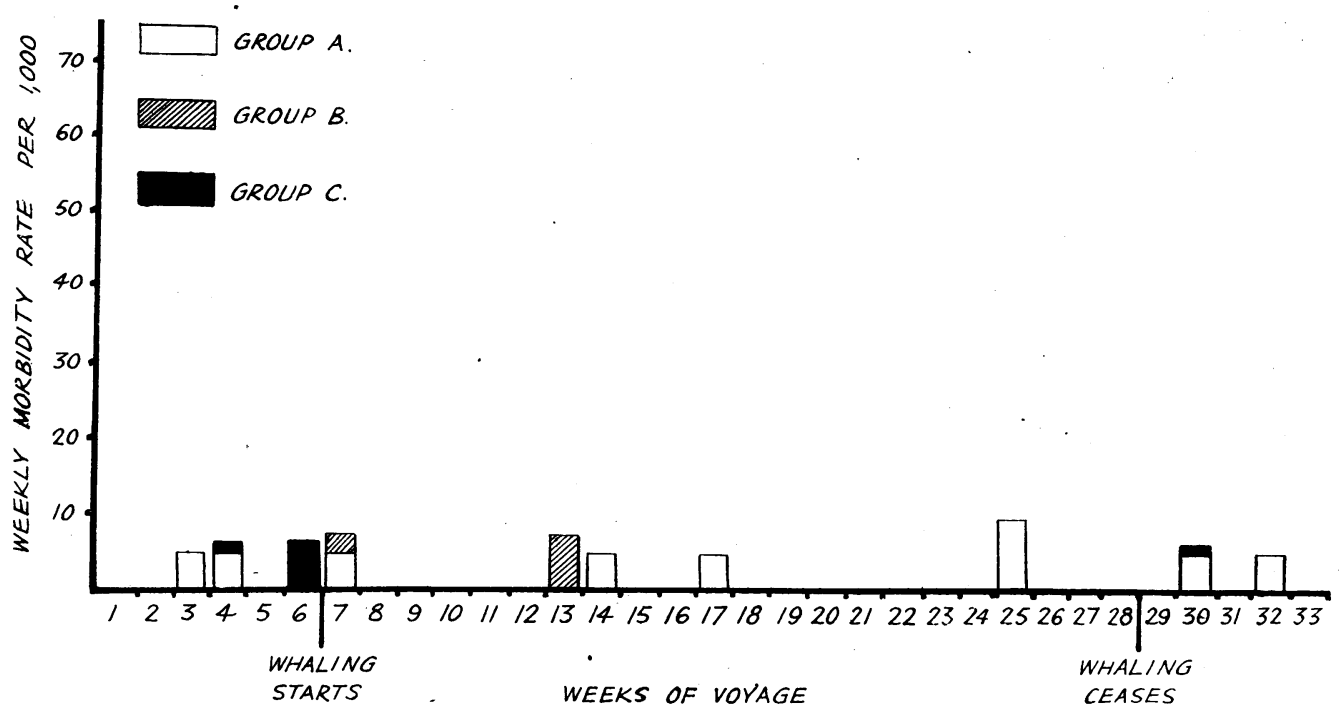

FIG. 13.-Weekly morbidity rate per 1,000 for each group for Column 13 (diarrhoea).

computations because the difficulty of reporting for the more trivial ailments might bias the results.

The mean of the differences is taken as the fairest estimate because the values are all true paired values; all adventitious factors such as climatic conditions, the number of whales taken and their degree of decomposition will be exactly the same for each group. The parameters of significance are given.

\section{Discussion}

During the voyage 933 reports of sickness were made (a total morbidity rate of 1,728 per thousand) and two deaths occurred (a mortality rate of 3.7 per thousand). Owing to the possibility of double entry previously mentioned, the total appearing in Table IV is 1,026. This will only bias the columns total morbidity and total classified morbidity, and these only to a small extent.

Tables IV and V are included mainly to provide the data on which the statistical operations were conducted, and to record this data for the convenience of future workers in this field.

Table VI shows that the whaling season has a significant influence upon the total weekly morbidity rate per thousand, and that this influence is most marked in group A. (Difference $=+63 \cdot 3$, " $t$ " $=$ $4 \cdot 0, P<0 \cdot 001$ for group $A$; difference $=+9 \cdot 7$, " $t$ " $=0.8, \quad P=0.4$ for group $B$; difference $=$ $+26 \cdot 7, " t "=2 \cdot 9, P=0.007$ for all three groups compounded.) These estimates might be slightly biased by the possibility of double entry.

When the columns are considered individually (ignoring column 14, Miscellaneous conditions, which is too heterogeneous a classification for analysis), there is seen to be an increased mean weekly morbidity rate per thousand in nine of the thirteen columns of group A during the whaling 의 period. In five of these cases the increase is $D$ statistically significant if the value $P=0.05$ is

TABLE VII

The Influence of Occupátional Group on Disease: Groups A and B

\begin{tabular}{|c|c|c|c|c|c|c|c|c|c|c|c|c|c|c|c|}
\hline Column & & 1 & 2 & 3 & 4 & 5 & .6 & 7 & 8 & 9 & 10 & 11 & 12 & 13 & Total \\
\hline Transit & $\begin{array}{l}d \\
t \\
p\end{array}$ & $\begin{array}{c}-2 \cdot 1 \\
1 \cdot 0 \\
0.35\end{array}$ & $\begin{aligned}-1.9 \\
0.7 \\
0.50\end{aligned}$ & $\begin{array}{l}+3.5 \\
0.9 \\
0.40\end{array}$ & $\mid \begin{array}{c}-1 \cdot 2 \\
0.6 \\
0.55\end{array}$ & $\begin{array}{c}+0.2 \\
0.1 \\
0.90\end{array}$ & $\begin{array}{c}-0.5 \\
0.4 \\
0.70\end{array}$ & $\begin{array}{c}+1.9 \\
1.0 \\
0.35\end{array}$ & $\begin{array}{c}+3.5 \\
1.7 \\
0.15\end{array}$ & $\mid \begin{array}{l}+0.9 \\
0.8 \\
0.45\end{array}$ & $\begin{array}{c}+0.3 \\
0.2 \\
0.80\end{array}$ & $\mid \begin{array}{c}1 \cdot 2 \\
1.9 \\
0 \cdot 10\end{array}$ & $\begin{array}{c}+0.2 \\
0.4 \\
0.70\end{array}$ & $\left|\begin{array}{c}+1.6 \\
2.3 \\
0.055\end{array}\right|$ & $\begin{array}{r}3.7 \\
0.4 \\
0.70\end{array}$ \\
\hline Whaling & $\begin{array}{l}\mathbf{d} \\
\mathbf{t} \\
\mathbf{P}\end{array}$ & \begin{tabular}{|c|}
+13.3 \\
4.8 \\
$<0.001$
\end{tabular} & $\begin{array}{c}+10.5 \\
3.7 \\
<0.001\end{array}$ & $\begin{array}{c}+9.8 \\
4.9 \\
0.001\end{array}$ & $\begin{array}{c}+4.7 \\
2.5 \\
0.02\end{array}$ & $\begin{array}{c}+5.5 \\
2.8 \\
0.019\end{array}$ & $\begin{array}{r}+2.4 \\
1.5 \\
0.15\end{array}$ & \begin{tabular}{|c|}
+2.9 \\
2.3 \\
0.035
\end{tabular} & $\begin{array}{c}+1.3 \\
1.6 \\
0.13\end{array}$ & $\begin{array}{c}-0.2 \\
0.1 \\
0.90\end{array}$ & $\begin{array}{c}-1 \cdot 3 \\
1 \cdot 1 \\
0.27\end{array}$ & $\mid \begin{array}{c}+2 \cdot 2 \\
1 \cdot 7 \\
0 \cdot 10\end{array}$ & $\mid \begin{array}{c}+0.1 \\
0.1 \\
0.90\end{array}$ & $\begin{array}{c}+0.4 \\
0.6 \\
0.55\end{array}$ & $\begin{array}{r}+53.3 \\
7.5 \\
<0.00\end{array}$ \\
\hline
\end{tabular}

$d=$ mean of differences of weekly morbidity rate per 1,000 of groups A and B.

n transit $=11$
nhaling $=22$

$A+$ means a larger $\mathbf{A}$ value. 
accepted as the criterion of significance. In group B there is an increase in eight cases, but this increase does not attain statistically significant proportions.

Table VII shows that during the transit period group. A had a higher morbidity rate than group B in nine columns, but this difference never assumed significant proportions, and only approached significance in one case. During the whaling period group $\mathbf{A}$ had a greater morbidity in all thirteen columns, and in six cases this difference was significant.

It may be fairly concluded from the above that whaling exerted a deleterious influence upon the health of the expedition's complement, and that this influence was felt almost entirely by the deck and factory workers, that is, by those actually handling the whale carcases. This influence was most marked in certain disease categories, viz. suppurative lesions; traumatic lesions without sepsis; nondiarrhoeic alimentary disorders; and dental conditions.

The increase in traumatic lesions might be expected as a consequence of the increased dangerous activity during the whaling period, and could be expected to contribute in some measure to the increase of sepsis, but the proportion of septic lesions to traumatic lesions without sepsis also rises.

The suppurative lesions may reasonably be assumed to have a relationship to the bacteriological findings discussed later, though no rigid establishment of causality by bacteriological methods was possible.

Some dried cultures from wounds were brought back on board Balaena and were examined after a lapse of about six to eight months, but only nonpathogenic clostridia and staphylococci were grown (Hobbs, 1947). However, a duplicate of a preparation that had previously shown $\mathrm{Cl}$. oedematiens when flown back (Parish, 1947) was opened and examined at the same time as the preparations from septic wounds, and failed to reveal any pathogenic clostridia. It seems highly likely that some of the bacterial strains originally present were no longer viable after the time which had elapsed since drying. The drying was carried out on a vacuum pump, but no preliminary freezing technique was used.

Whether the increased incidence of alimentary disorders, "rheumatic" conditions, and dental disorders bear any relationship to the bacterial hazard or not is not shown.

The alimentary disorders were mainly a form of "gastritis" and this might have been produced partly by continuous reflex stimulation due to the gases from decomposing protein. It seems to be generally supposed that the smell of a whale-factory is due to the boiling of fatty material. In fact it seems very largely due to the anaerobic bacterial decomposition of hundreds of tons of meat (one whale may weigh over one hundred tons, of which approximately 70 per cent. is said to be muscle, and the flesh of three whales may easily be on deck at one time), and is present continuously. The smell is certainly extremely nauseating, and very little olfactory fatigue sets in.

The sepsis occurring was of great interest. In the two instances of death, post-mortem examinations were conducted, and extracts from the reports of these are given below.

\section{Case 1}

The victim, a plan foreman, was struck on the head by a hook when a cable parted. Death occurred two days later.

"Death was due to acute meningitis and cerebral oedema following a fracture of the skull due to direct violence. A contributory factor was bronchopneumonia. Whilst it is impossible to say whether the trauma alone would have proved fatal, there can be little doubt that the chances of survival were seriously jeopardized by the spread of infection to the meninges. The bacterial flora found was similar to that recovered from the muscle of ' burned ' (decomposed) whale." (Sporing Gram-positive bacilli, Gram-negative bacilli, and streptococci.)

The whale that was being handled at the time was a very decomposed fender whale, and the whole of the musculature was reduced to a greyish putty-like mass by putrefaction.

\section{Case 2}

A deck hand who ran a steel hook into his calf died under anaesthesia during an operation for amputation of the leg for gas gangrene. This operation was started forty-eight hours after the accident. Penicillin and sulphonamide had been given. Clinically the case was a typical one of fulminating gas gangrene.

" The appearances are those of an acute toxaemia from fulminating gas gangrene. Though it is impossible to exclude the anaesthetic as a factor in accelerating death, the amount of thrombus in the heart indicates that the patient was in fact moribund at the time the operation was commenced. The source of the infection was the wound of the right leg."

Bacteriological examination revealed the presence of a sporing Gram-positive bacillus only.

Thus in one of these cases death was indisputably due to gas gangrene, and sepsis that could have originated in whale muscle cannot be excluded as a factor causing death in the other.

Many other cases were very resistant to treatment, and some were clinically gas-forming infections.

During the flensing and subsequent operations it is very common for the workers to acquire small cuts on their hands, or to pierce their hands or legs with hooks or wires. The danger from these 
wounds is well recognized in whaling lore, the resultant intractable and often crippling sepsis being spoken of generally as " blubber-finger." It is said that the incidence and severity of this condition variess from year to year and even from region to region in the same year. Tales are told of ships losing many men in a season from this complaint, but little medical investigation of the condition seems to have been undertaken.

It is also said that a " sick whale "' is particularly likely to cause such trouble, but it was impossible to find out exactly what a " sick whale" is.

Some bacteriological studies had been carried out on board Balaena during the season (Case, 1947), and it had been established that, quite soon after the death of the whale, the musculature became heavily contaminated with a mixed bacterial flora derived presumably from the intestinal tract. This flora consisted typically of sporing and non-sporing Gram-positive bacilli, proteolytic, saccharolytic, and sometimes both; Gram-negative bacilli; and faecal-type streptococci. Occasionally, but not typically, Staphylococcus albus was found. The bacterial contamination became progressively more massive as the time from death increased.

It was also established that the temperature of the whale in vivo was about $35^{\circ} \mathrm{C}$., and that this temperature was maintained to within a degree even in a whale that had been left in the sea for five or six days after death. It was therefore not surprising to find that rapid invasion of the musculature took place.

Dried cultures of bacteria grown from whale muscle had been sent back to this country by air, and, amongst a mixed flora of clostridia, $\mathrm{Cl}$. oedematiens (Parish, 1947) and Cl. chauvoei (Shrewsbury, 1947) were identified.

Swabs were taken from a series of cases of sepsis, and cultured in peptone water and cooked-meat medium. Direct smears were also examined. The findings are shown in Table VIII.

It is seen that the three main types of bacteria found in decomposing whale muscle and in the contents of the whale's intestine appeared frequently in these wounds. The most notable exception was

TABLE VIII

Bacteriological Findings in Fifteen Cases of Sepsis (Direct film and culture)

\begin{tabular}{|c|c|c|c|c|c|c|c|c|}
\hline Period & Group & \multicolumn{3}{|c|}{ Nature of wound } & $\mathbf{G}+$ ve $B$ & $G-v e B$ & Strep & Staph \\
\hline $\mathbf{W}$ & $\mathbf{A}$ & Suppurative otitis media & . & . & + & + & + & + \\
\hline $\mathbf{W}$ & $\mathbf{A}$ & Septic laceration of hand & .. & . & + & + & + & + \\
\hline W & $\mathbf{A}$ & Fatal puncture wound of leg & .. & . & + & - & - & - \\
\hline $\mathbf{W}$ & A & Fatal wound of head & . & . & + & + & + & - \\
\hline W & $\mathbf{A}$ & Septic laceration of hand & .. & . & + & - & + & - \\
\hline $\mathbf{w}$ & $\mathbf{A}$ & \multicolumn{2}{|c|}{ Septic traumatic amputation of finger } & . & + & - & - & - \\
\hline $\mathbf{W}$ & A & Septic finger .. $\quad$. & .. & . & + & + & + & + \\
\hline $\mathbf{w}$ & $\mathbf{A}$ & \multicolumn{2}{|c|}{ Urethral smear (non-specific urethritis) } & $\cdot$ & + & + & - & + \\
\hline $\mathbf{w}$ & $\mathbf{A}$ & Septic finger $\ldots \quad \ldots$ & .. & .. & + & - & - & + \\
\hline $\mathbf{W}$ & B & Septic laceration of hand & .. & .. & + & + & + & - \\
\hline $\mathbf{W}$ & B & Septic laceration of hand & .. & . & + & + & + & + \\
\hline W & B & Septic finger .. & .. & .. & + & + & + & - \\
\hline $\mathbf{W}$ & $\mathbf{C}$ & Septic finger .. $\quad .$. & $\cdots$ & .. & - & - & - & + \\
\hline $\mathbf{w}$ & C & Septic laceration of hand & .. & .. & - & 一 & - & + \\
\hline $\mathbf{W}$ & $\mathrm{C}$ & Septic laceration of hand & .. & .. & - & - & 一 & + \\
\hline
\end{tabular}


in the three cases of catchers' crew patients, where staphylococcus only was found. All the other cases showed Gram-positive bacilli. In some of the cases these were probably saprophytic, in others they were probably pathogenic, and in one case the cause of death.

The heavy bacterial contamination of much of the material handled by the deck workers, under conditions where wounds are common, presents an obvious hazard; and it is suggested that the increased sepsis rate amongst deck workers is probably caused by this contamination. In view of the actual occurrence of gas-forming infections, the findings of pathogenic clostridia of the gasgangrene group in cultures from whale-muscle, and the reports of high mortality rates in some years from what sounds like a type of gas gangrene, it is felt that the risk of clostridial infection as a hazard of whaling should be borne in mind by the ships' medical officers, and that appropriate hospital stores should be carried.

The risk of sepsis might be mitigated to some extent by keeping the period between the death of the whale and processing as short as possible, and by refraining from flensing decomposed fender whales, or, as sometimes happens, whales that are found drifting about dead and decomposed. These whales are presumably " flagged" whales that have been lost. Cleansing of the decks and cleaning of hooks in antiseptics might also prove desirable.

The possibility of seasonal and regional variations of the predominating bacterial flora, which is presumably derived from the Euphausids and squids on which the baleen and toothed whales respectively feed, should be considered. It would seem highly desirable for further bacteriological studies of sepsis to be carried out.

The possible implications of bacterial contamination of whale meat intended for human consumption are, and have been for some time, under consideration by competent authorities in this country, and none of the findings reported in this paper should be interpreted as suggesting that such meat as is taken for human consumption is necessarily in any way unfit or suspect.

\section{SUMMARY}

The hospital data accumulated on a whaling expedition to the Antarctic pelagic whaling grounds is analysed and discussed, and it is shown that the whaling season exercises a deleterious influence on the health of the crew of the factory ship.

This influence is felt in the main by the deck workers and factory workers, and the largest increase of disease occurs in the diseases classified as suppurative lesions, traumatic lesions without sepsis, non-diarrhoeic alimentary disorders, " rheumatic" conditions, and dental disorders.

The sepsis, which in this cruise caused one death and was at least a contributory factor in the second, was often associated with the typical bacterial flora of the whale's intestine, which soon after death causes heavy bacterial contamination of the whale muscle. It is suggested that gas gangrene should be thought of as a real danger to whalers, and that the hospital stores and equipment should be organized accordingly.

The figures are given in detail, in order to form a body of data for reference for other workers in this field.

It is suggested that every endeavour should be made to keep down the time elapsing between killing and processing the whale, and that the practice of flensing fender whales after they have become decomposed is very undesirable from a hygienic point of view.

My thanks are due to Dr. Jamis Hutchison, Medical Officer on board Fl. F. Balaena 1946-7, for all the co-operation he gave in making it possible to complete these studies, to Mr. Kenneth Whittle of the Royal Isle of Wight County Hospital, and to Mr. Alexander Ireland, of the Infirmary for Sick Children, Glasgow, for their invaluable help in the laboratory investigations on board.

My thanks are also due to Professor J. D. Shrewsbury, the University of Birmingham, Dr. Betty Hobbs, Central Public Health Laboratory, and Dr. H. J. Parish, of Messrs. Burroughs Wellcome Ltd., for examining bacteriological material brought back, and, in the case of Dr. Parish, for advising upon and obtaining prophylactic and therapeutic sera for the next expedition. United Whalers Ltd. have carried out part of the recommendations outlined above.

\section{REFERENCES}

Case, R. A. M. (1947). Station Record Memorandum (Unpublished) No. 217 of the Low Temperature Research Station, Cambridge.

Hobbs, Betty C. (1947). Personal communication to the author.

Parish, H. J. (1947). Report to the Low Temperature Research Station, Cambridge.

Shrewsbury, J. F. D. (1947). Personal communication to the author.

“ Student", (1908). Biometrika, 6. 1 